2017-03

\title{
Current and wave effects around windfarm monopile foundations
}

\author{
Miles, Jonathon
}

http://hdl.handle.net/10026.1/8561

10.1016/j.coastaleng.2017.01.003

Coastal Engineering

Elsevier BV

All content in PEARL is protected by copyright law. Author manuscripts are made available in accordance with publisher policies. Please cite only the published version using the details provided on the item record or document. In the absence of an open licence (e.g. Creative Commons), permissions for further reuse of content should be sought from the publisher or author. 


\title{
Current and wave effects around windfarm monopile foundations
}

\author{
Jon Miles $^{\mathrm{a} \dagger}$, Tom Martin ${ }^{\mathrm{a}}$, Liam Goddard ${ }^{\mathrm{a}}$ \\ ${ }^{a}$ School of Marine Science and Engineering, Plymouth University, Plymouth, PLA 8AA, UK. \\ ${ }^{\dagger}$ Corresponding author: Tel: +44 1752 584737; Fax: +44 1752 586101; email: \\ jrmiles@plymouth.ac.uk.
}

Note: This is the author's version of a paper published in Coastal Engineering. Please download the original Coastal Engineering paper and reference it as: Miles, J., Martin, T., and Goddard, L., 2017. Current and wave effects around windfarm monopile foundations. Coastal Engineering 121, 167-178, Doi 10.106/j.coastaleng.2017.01.003

\begin{abstract}
Laboratory measurements were undertaken to investigate wave and current velocities in the vicinity of a wind turbine monopile foundation, in order to inform environmental impact assessments and to quantify flow variability in the region of the power take off cable. Flow measurements were made up to 15.5 pile diameters (D) downstream of the pile.

Measurements were also taken around the perimeter of the pile ( 0.75 D from the pile centre) at the approximate representative height of the power cable.

In current-only tests, the mean flow was reduced immediately downstream of the pile, but returned to within $5 \%$ of background levels by $8.3 \mathrm{D}$ downstream of the pile centre in representative conditions. A new parameterisation of the velocity recovery is given. The turbulent eddy shedding frequency was well predicted by the Strouhal number. Turbulence peaked at $1.5 \mathrm{D}$ from the pile, and the subsequent decay was parameterised. Velocity magnitudes at the side of the pile were up to 1.35 times greater than background flow rates, in line with potential flow theory. Velocities in the wake region were much less than predicted by potential flow theory, corresponding with increased turbulence.
\end{abstract}

Tests with waves indicated that oscillatory velocities reduced immediately down-wave of the pile, but returned quickly to background levels (by 1.65 to $3.5 \mathrm{D}$ of the pile centre). The general near-pile distribution of the orbital velocity maximum was well represented by potential flow theory. Orbital velocities were reduced immediately up-wave and down-wave of the pile. At the side of the pile in wind sea conditions, the velocity increased up to 1.66 times the background level. This increased to 1.85 times in swell conditions.

For orthogonal currents and waves, a velocity parameter was calculated as the mean current plus wave orbital velocity, resolved. With the mean current direction as a reference, the maximum flow was observed at the side of the pile. At $0.75 \mathrm{D}$ from the pile centre, the flow was enhanced by up to 1.2 times the no-pile case. Spectral peaks in the velocity were evident at both wave frequency and at the Strouhal frequency, immediately down current from the pile.

Keywords: marine renewable energy; offshore windfarms; monopile foundations; current measurements; environmental impact. 


\section{Introduction}

Offshore wind turbines have become an important contributor to renewable energy generation. The foundations of monopiles are known to modify flows, and generate complex three dimensional localised flow structures (Sumer et al., 1997; Roulund et al., 2005; Petersen et al., 2015). The pileinduced flow modifications affect scour generation, need to be considered for cable design, and may impact on local ecosystems. Flows passing a vertical cylinder are accelerated as they pass around it. This flow enhancement may be demonstrated theoretically by potential flow models (e.g. Acheson, 1990; White, 1991). In these models, the flow in the radial direction $U_{r}$ is given by:

$U_{r}=U_{\infty}\left(1-\frac{R^{2}}{r^{2}}\right) \cos \theta$

where $U_{\infty}$ is the flow strength away from the pile's influence, $R$ is the radius of the pile, $\mathrm{r}$ is the distance from the pile centre, and $\theta$ is the angle around the pile from the upstream stagnation point. The flow in the tangential direction $U_{\theta}$ is given by:

$U_{\theta}=-U_{\infty}\left(1+\frac{R^{2}}{r^{2}}\right) \sin \theta$

The magnitude of the flow is given by:

$U=\left(U_{r}{ }^{2}+U_{\theta}{ }^{2}\right)^{1 / 2}$

These predictions do not include the generation of vortices, or turbulence in the wake region, and may therefore misrepresent the flow. For offshore monopiles, three key mechanisms of turbulence generation are recognised (Petersen et al., 2015). These include lee wake vortices, horseshoe vortices and (vertical) counter-rotating vortices (Fig. 1).

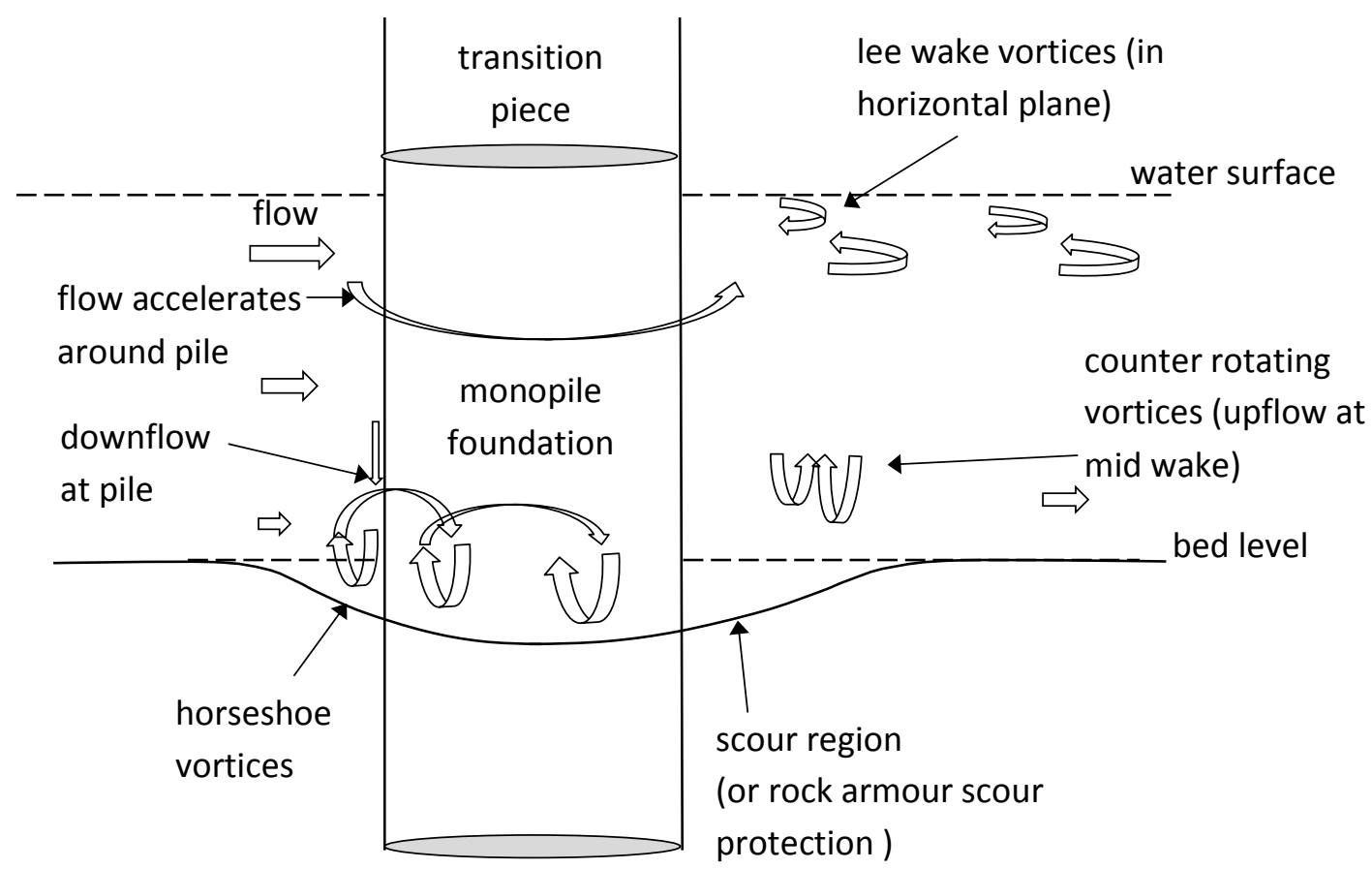

Fig. 1. Flow processes near windfarm monopile foundations. 
Lee-wake vortices are well documented (e.g. Acheson, 1990). They may be symmetrical and stationary at very low Reynolds number (Re around 30), or shed as vortex pairs in a von Karman vortex street at higher Reynolds number. For Re $>2000$, the wake is essentially turbulent, but still has traces of periodic structure remaining. The frequency of eddy shedding $\left(f_{s}\right)$ may be predicted using the Strouhal number $(S)$, the flow velocity away from the pile $\left(\mathrm{U}_{\infty}\right)$ and the pile diameter $(\mathrm{D})$ as:

$f_{S}=\frac{S U_{\infty}}{D}$

The Strouhal number $(S)$ is a function of the pile Reynolds number $\left(\operatorname{Re}_{\mathrm{D}}\right)$ :

$S=0.198\left(1-\frac{19.7}{R e_{D}}\right)$

The pile Reynolds number varies with flow velocity, pile diameter and fluid viscosity $v$ :

$R e_{D}=\frac{U_{\infty} D}{v}$

Horseshoe vortices are formed near the bed. These are generated by a downflow on the upstream side of the pile. The horseshoe vortices affect the region around the perimeter of the pile, near the bed (Baker, 1979; Dargahi, 1989; Sumer et al., 1997).

Counter rotating vortices in the vertical plane, downstream of the pile were observed to form in models with scour protection by Petersen et al. (2015). These give a net upward directed flow in a line downstream of the centre of the pile, in the lower part of the water column, balanced by a downward flow to the sides of the centreline.

In oscillatory flows, the Keulegan-Carpenter $(\mathrm{KC})$ number gives a measure of the orbital excursion compared to pile diameter (Sumer et al., 1997). The KC number is given by:

$$
K C=\frac{U_{m} T}{D}
$$

where $u_{m}$ is the maximum orbital velocity calculated from linear theory. The importance of flow separation increases with the KC number. Sumer et al. (1997) identify that for wave cases with KC < 6 , no horseshoe vortex occurs. With increasing KC, the size and lifespan of the horseshoe vortex increases. The importance of diffraction increases with the ratio D/ $\lambda$ (Sarpkaya and Isaacson, 1981). As $\mathrm{KC}$ increases, non-linear effects become important at gradually lower values of $\mathrm{D} / \lambda$.

The interaction between the flow and bed material leads to enhanced bed shear stress and the formation of scour around the pile base (Sumer et al., 1992; Sumer et al., 2001). The depth of scour has been observed to be up to 1.4 pile diameters in the field (Whitehouse et al., 2008), although there is variability depending on the sediment type (see Whitehouse et al., 2011). Scour may lead to concerns about structure stability, and in some cases, rock armour scour protection is put in place. When applied, the horizontal extent of this scour protection typically has a diameter of $2 \mathrm{D}$ to $4.5 \mathrm{D}$ (May et al., 2002; Matutanto et al., 2014).

Flow velocity has been shown in numerical models to be modified further downstream than is indicated by the size of the scour hole (Rivier et al., 2014; Rivier et al., 2016). Rogan et al. (2014) and Rogan et al. (2016) give a detailed study of the downstream impacts of turbulence, indicating that pile induced turbulence can remain for up to 80 pile diameters downstream of the pile. The change in mean flow may lead to larger scale variations in bedforms, as has been observed in the field at Scroby Sands windfarm, UK (Rees, 2006). 
The design of monopile foundations is such that power cables are routed to the seafloor near the base of the pile. There are different design approaches for the cabling, depending on whether scour protection is applied, and the specific design preferences of the installer (Carter, 2007; Vattenfall, 2011). The cable may be routed through a 'J-tube' that brings the cables to the structure base on the outside of the pile. Alternatively, cables may be routed inside the foundations, and leave through a port in the base of the foundation. Each monopile may require one or two cables, depending on the cable layout of the windfarm. The cable typically leaves the pile above the level of the bed, at about $\sim 2 \mathrm{~m}$ above undisturbed sand level and at a downward angle of $\sim 45^{\circ}$. This structure takes the cables across the deepest scour region (E.ON, pers comm). The diameter of the cable is small compared to the pile diameter (e.g. the inter-pile cable diameter is $\sim 13.9 \mathrm{~cm}$ for the UK Rampion wind site). Beyond the J-tube/port, a free hanging section of cable (catenary section) crosses the remaining scour region, and is then routed into a trench in the seafloor (substrate permitting) (E.ON, pers. comm.). The free section of the catenary cable is susceptible to hydrodynamic forcing from different sources, including loading from the mean flow, variability in loading due to monopile foundation induced turbulence, and vortex induced vibration of the cable itself. Furthermore, surface wind wave and swell driven oscillations may affect the flow velocity near the cable. The time varying nature of the flow gives rise to the potential for cyclic stress fatigue and cable failure.

Predictions of tidal currents and wave orbital velocities may be obtained on a large scale from oceanographic measurements and model predictions. However, the local modification of flow, as a result of the inclusion of the pile in the system, means that these velocity predictions do not necessarily accurately represent the velocity near the pile. Numerical models (Rivier et al., 2016) and laboratory experiments (Rogan et al., 2016) have illustrated that the monopile leads to considerable modification of parameters such as the near-pile velocity and bed shear stress. This paper documents new laboratory measurements of currents and wave orbital velocities in the vicinity of a model monopile and downstream of the pile, with the aim of offering guidance parameters describing flow modifications. This is necessary in order to inform both environmental impact assessment and cable design.

\section{Method}

\subsection{The COAST Basin}

Physical model experiments were carried out in the Plymouth University (UK) COAST basin. The basin is $15.5 \mathrm{~m}$ long, $10 \mathrm{~m}$ wide and $0.5 \mathrm{~m}$ deep. Pre-installed internally threaded anchor points on the basin floor allowed a scale model monopile to be securely attached. Fig. 2 shows the general basin set up during the experiments. The dimensions of the basin components are indicated in Fig. 3 .

An integral pump system generates currents across the basin. Pumped flow upwells through basin floor grids into a narrow (turbulent) pool. The currents were driven through a flow straightener block at the upstream side of the basin, to reduce turbulence in the flow as far as possible. The block was formed from multiple parallel pipes that were horizontal and in-line with the flow. The individual pipes were approximately circular, of diameter $12 \mathrm{~mm}$, and length $0.3 \mathrm{~m}$. The straightener block extended over the full water depth $(0.5 \mathrm{~m})$. The pump driven flows were calibrated using Acoustic Doppler Velocimeter (ADV) measurements.

Waves were generated using a bank of full depth wave generators located at one end of the basin. The paddles were piston driven and designed to give equal horizontal displacement over depth at the point of generation. Waves in this experiment were set to a sinusoidal profile. Wave heights were calibrated 
using a resistance wire wave gauge. In the wave plus current tests, the wave direction was orthogonal to the current direction. After passing the monopile test area, wave energy was absorbed by a solid linear sloping beach (slope 1 in 10).

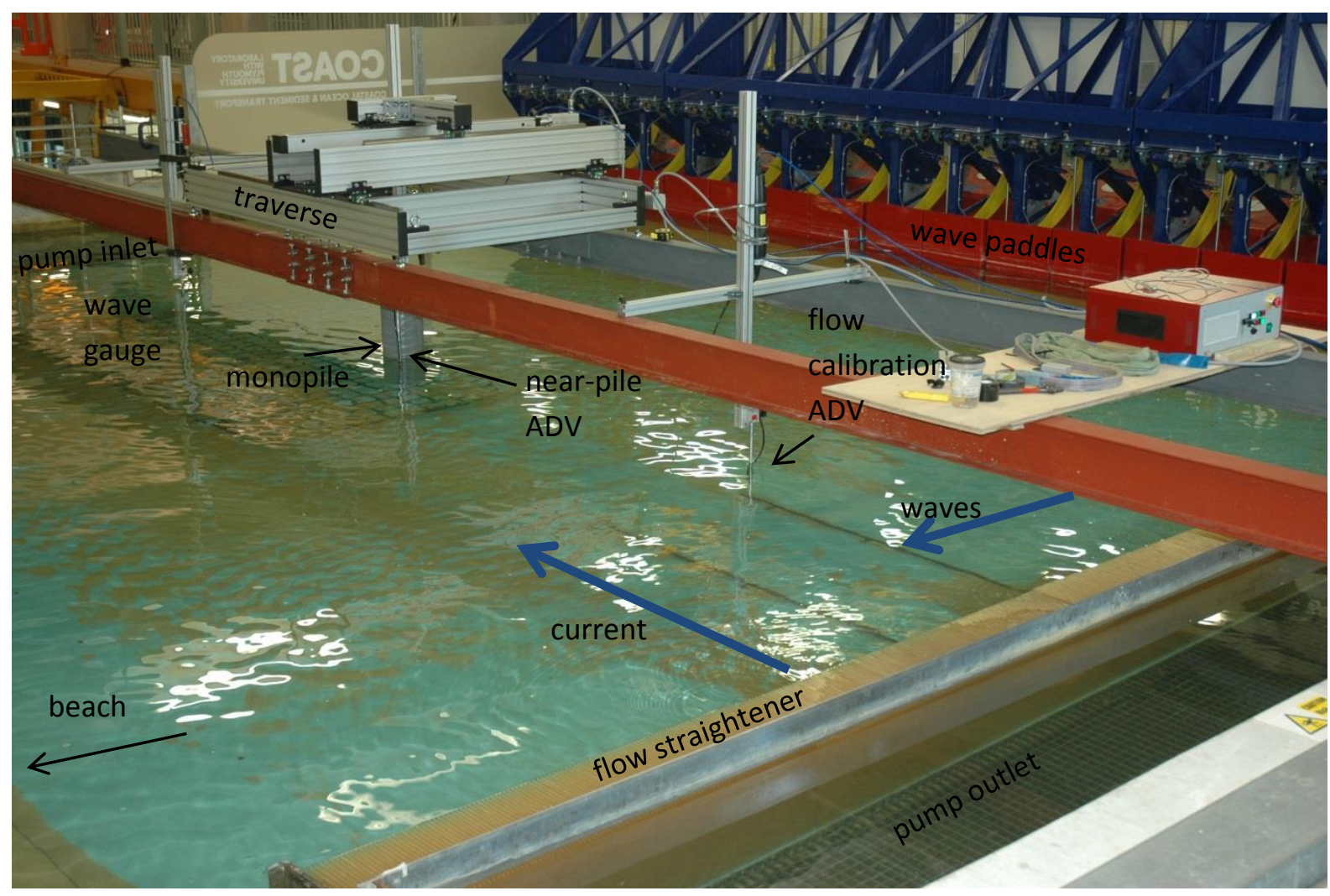

Fig. 2. Photo shows the model monopile (grey cylinder), current outflow from recirculating pumps, flow straightening block, instrument traverse, wave paddles, ADVs for flow calibration and measurement, and wave gauge. The sloping beach is to the immediate left of the photo.

High frequency current measurements were made using a Nortek Vectrino Profiler Acoustic Doppler Velocimeter (ADV) (Fig. 4). The Vectrino was placed in a downward looking orientation, giving a velocity profile with 32 measurement bins over a $3.5 \mathrm{~cm}$ range. The instrument was located carefully so that the bed was identifiable in the lowest $0.5 \mathrm{~cm}$ of the measurement range. The water in the basin was seeded to maximize signal amplitude. Data was logged at $64 \mathrm{~Hz}$, with the aim of high resolution velocity measurements for waves of period $1.25 \mathrm{~s}$ and $2 \mathrm{~s}$. Data was recorded for 5 minute runs, to give approximately 200 or 150 waves in each record respectively.

\subsection{Prototype field parameters}

Conditions at existing windfarm sites off the UK coast are identified in various reports, including CEFAS (2006), Whitehouse et al. (2011), Matutanto et al. (2014), various websites (4COffshore.com, LORC.dk) and UK Admiralty Charts. North Sea and Irish Sea windfarms are located in a variety of water depths from $2 \mathrm{~m}$ deep sandbanks (e.g. Gunfleet Sands) to $34 \mathrm{~m}$ (Greater Gabbard). Monopile diameters (D) in the North Sea and Irish Sea windfarms in the British Sector vary from $4 \mathrm{~m}$ (North Hoyle) to $6.5 \mathrm{~m}$ (Walney Phase II). Tidal current speeds are site dependent, with typical rates in the region $0.9 \mathrm{~m} / \mathrm{s}$ at Kentish Flats (Whitehouse et al., 2011), to $1.4 \mathrm{~m} / \mathrm{s}$ at Scroby Sands (CEFAS, 2006). 
Wave periods (T) are typically in the region 6 to 10 seconds for sea and swell conditions respectively. Significant wave heights $(\mathrm{H})$ are typically in the region of $1 \mathrm{~m}$ to $2 \mathrm{~m}$. Storm conditions of up to $2.1 \mathrm{~m}$ were recorded at Scroby Sands (CEFAS, 2006), but were limited by wave breaking.

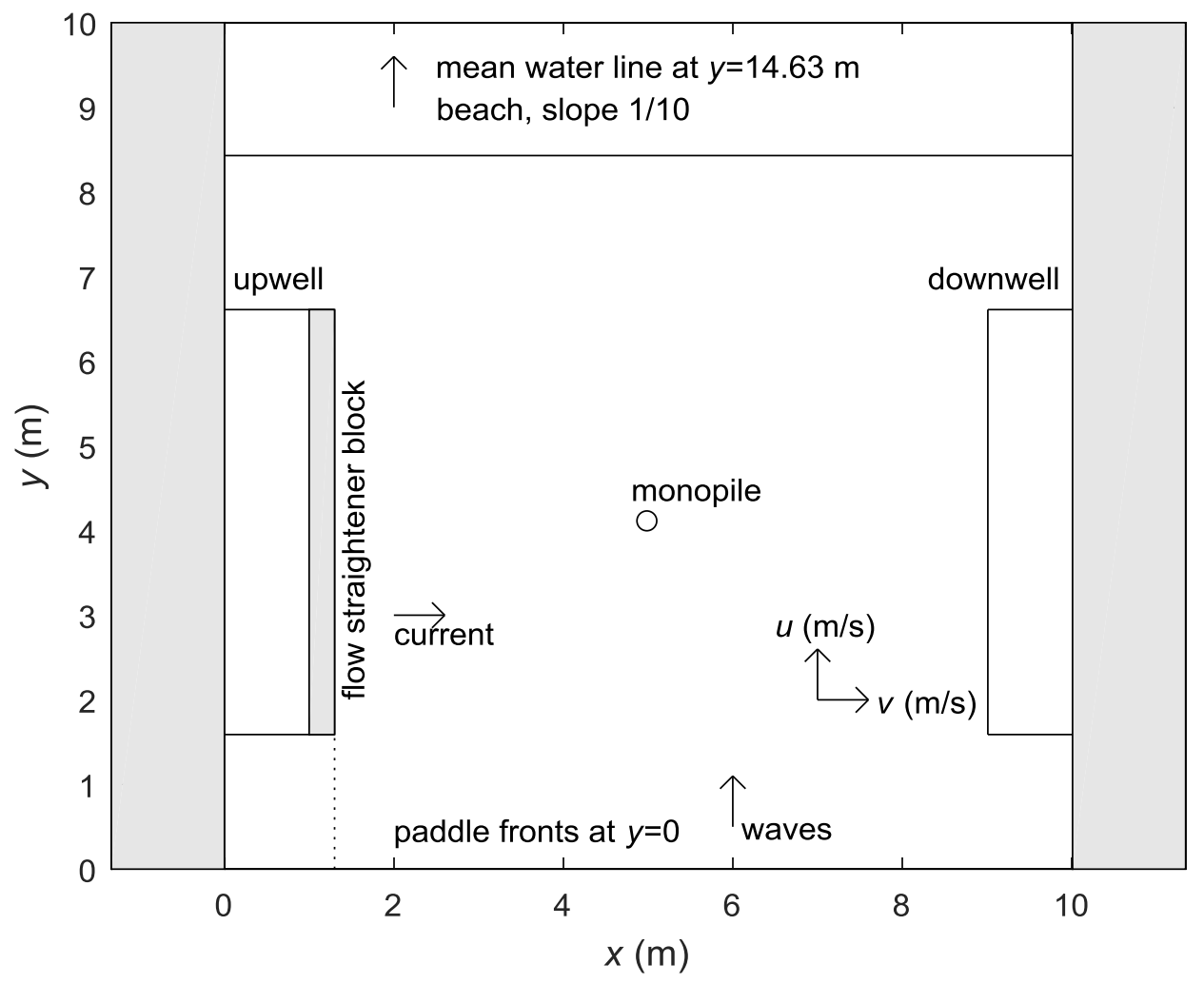

Fig. 3. Plan view of basin layout indicating basin dimensions, monopile position, current outflow region, flow straightening block position, wave paddles and sloping beach.

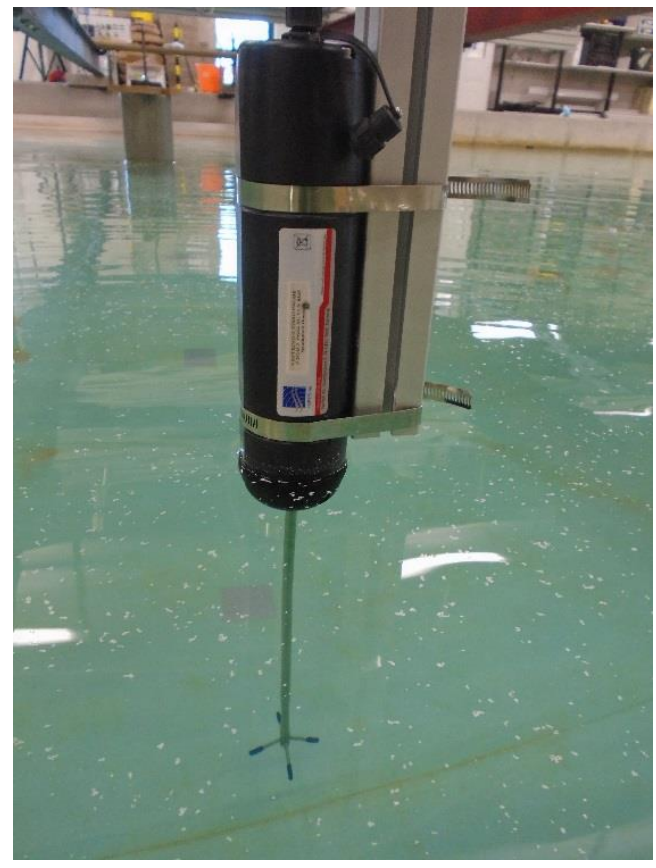

Fig. 4. Photo showing Vectrino Profiler ADV, with model monopile visible in the background. 
Due to the large differences between sites, a 'representative case' set of conditions for the experiments described here was assumed, with a pile diameter of $5 \mathrm{~m}$, water depth of $12.5 \mathrm{~m}$, current speed (U) of $1.25 \mathrm{~m} / \mathrm{s}$. Wave parameters are specified nominally as typical 'windsea' conditions, with $\mathrm{H}=1 \mathrm{~m}$ and $\mathrm{T}=6.25 \mathrm{~s}$. Tests were carried out with current only, wave only and orthogonal wave + current conditions. To extend the results beyond the representative case and explore trends, four further current conditions were considered, such that the total set of prototype current conditions was $\mathrm{U}=0.75 \mathrm{~m} / \mathrm{s}, 1 \mathrm{~m} / \mathrm{s}, 1.25 \mathrm{~m} / \mathrm{s}, 1.5 \mathrm{~m} / \mathrm{s}$ and $1.75 \mathrm{~m} / \mathrm{s}$. Two further wave cases were investigated, alongside the 'windsea' case $(H=1 \mathrm{~m}, \mathrm{~T}=6.25 \mathrm{~s})$. These are termed a 'swell' case $(H=1 \mathrm{~m}, \mathrm{~T}=10$ s) and a 'storm' case $(\mathrm{H}=2 \mathrm{~m}, \mathrm{~T}=6.25 \mathrm{~s})$.

\subsection{Model scaling}

The experiment scaling was governed by the physical characteristics of the available wave basin, as well as the prototype conditions relevant for testing. The basin depth was fixed at $0.5 \mathrm{~m}$. For a representative prototype water depth of $12.5 \mathrm{~m}$, a geometric scaling of 1:25 was possible. A prototype pile diameter of $5 \mathrm{~m}$ therefore scaled to a model pile diameter of $0.2 \mathrm{~m}$. Velocity and wave period were scaled according to Froude similitude. A parameter set to guide the experiments with appropriate current and wave period scaling is shown in the Table 1.

\begin{tabular}{lccc}
\hline & $\begin{array}{c}\text { Scaling } \\
(\mathrm{S}=1: 25)\end{array}$ & Prototype & Model \\
\hline Monopile Diameter (D) & $\mathrm{S}$ & $5 \mathrm{~m}$ & $0.2 \mathrm{~m}$ \\
\hline Water Depth & $\mathrm{S}$ & $12.5 \mathrm{~m}$ & $0.5 \mathrm{~m}$ \\
\hline Current Speed & $\mathrm{S}^{0.5}$ & $0.75 \mathrm{~m} / \mathrm{s}$ & $0.15 \mathrm{~m} / \mathrm{s}$ \\
Current Speed & $\mathrm{S}^{0.5}$ & $1.0 \mathrm{~m} / \mathrm{s}$ & $0.2 \mathrm{~m} / \mathrm{s}$ \\
Current Speed & $\mathrm{S}^{0.5}$ & $1.25 \mathrm{~m} / \mathrm{s}$ & $0.25 \mathrm{~m} / \mathrm{s}$ \\
Current Speed & $\mathrm{S}^{0.5}$ & $1.5 \mathrm{~m} / \mathrm{s}$ & $0.3 \mathrm{~m} / \mathrm{s}$ \\
Current Speed & $\mathrm{S}^{0.5}$ & $1.75 \mathrm{~m} / \mathrm{s}$ & $0.35 \mathrm{~m} / \mathrm{s}$ \\
\hline Windsea: Wave Height & $\mathrm{S}$ & $1 \mathrm{~m}$ & $0.04 \mathrm{~m}$ \\
Windsea: Wave Period & $\mathrm{S}^{0.5}$ & $6.25 \mathrm{~s}$ & $1.25 \mathrm{~s}$ \\
Windsea: KC & $1: 1$ & 0.70 & 0.70 \\
Windsea Wavelength $(\lambda)$ & $\mathrm{S}$ & $54.5 \mathrm{~m}$ & $2.18 \mathrm{~m}$ \\
Windsea D/ $\lambda$ & $1: 1$ & 0.09 & 0.09 \\
\hline Swell: Wave Height & $\mathrm{S}$ & $1 \mathrm{~m}$ & $0.04 \mathrm{~m}$ \\
Swell: Wave Period & $\mathrm{S}^{0.5}$ & $10 \mathrm{~s}$ & $2 \mathrm{~s}$ \\
Swell KC & $1: 1$ & 0.97 & 0.97 \\
Swell Wavelength $(\lambda)$ & $\mathrm{S}$ & $101.4 \mathrm{~m}$ & $4.05 \mathrm{~m}$ \\
Swell D/ $\lambda$ & $1: 1$ & 0.05 & 0.05 \\
\hline Storm: Wave Height (Storm) & $\mathrm{S}$ & $2 \mathrm{~m}$ & $0.08 \mathrm{~m}$ \\
Storm: Wave Period & $\mathrm{S}^{0.5}$ & $6.25 \mathrm{~s}$ & $1.25 \mathrm{~s}$ \\
Storm KC & $1: 1$ & 1.41 & 1.41 \\
Storm Wavelength $(\lambda)$ & $\mathrm{S}$ & $54.5 \mathrm{~m}$ & $2.18 \mathrm{~m}$ \\
Storm D/ $\lambda$ & $1: 1$ & 0.09 & 0.09 \\
\hline
\end{tabular}

Table 1. Parameter set guiding the experiments.

For the purpose of this study, it was assumed that a notional cable would pass a region $1.25 \mathrm{~m}$ away from the pile at a height of approximately $0.75 \mathrm{~m}$ from the bed, in line with advice from E.ON. In the scaled model, this region is $5 \mathrm{~cm}$ from the pile and $3 \mathrm{~cm}$ above the bed. This position is as close to the 
pile as possible with the downward looking ADV used, and is at a height above the bed that allows the bed and near-bed flow to be identifiable in the ADV output. For a $0.2 \mathrm{~m}$ pile, this position is 0.75 $\mathrm{D}$ from the centre of the pile. No physical model of a cable was included. This position in the model was used as a starting point for flow measurement. Data from further positions were then obtained to investigate variation in flow.

A computer controlled instrument traverse was employed to control the position of the current meter in the immediate vicinity of the pile. Instrument locations relative to the pile were checked carefully using a metal tape measure. When outside the region controlled by the traverse, the instrument was fixed in position using standard laboratory clamps.

The ADV was re-positioned (when necessary) for each run. The measurement positions occupied by the ADV are illustrated in Fig 5. The base of the basin was a smooth painted texture. A very thin layer of fine sand was present on the base of the tank during the experiments.

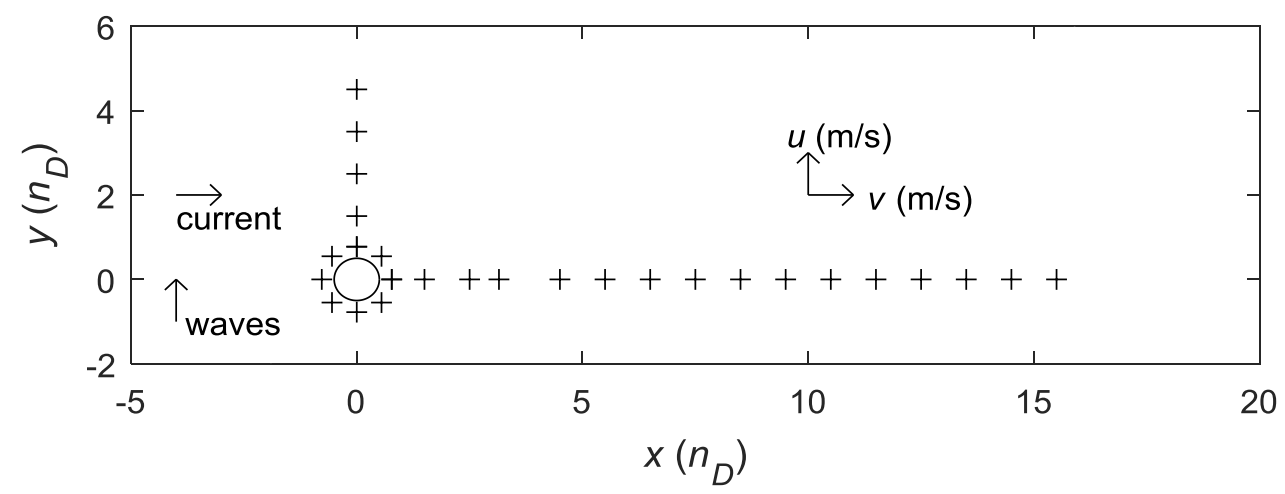

Fig. 5. Plan view of ADV measurement locations and axis definitions, in relation to the monopile. The pile diameter is $20 \mathrm{~cm}$. Distances are scaled by the number of pile diameters $\left(n_{D}\right)$. Measurements were made at the downstream position of 3.15 D instead of 3.5 D due to limited movement of the instrument traverse.

In the current only case, measurements were taken from a near-pile position, 0.75 pile diameters (D) from the centre ( $5 \mathrm{~cm}$ from the edge of the pile). Further measurements were made at $1.5 \mathrm{D}$ from the centre (i.e. $20 \mathrm{~cm}$ from the edge), and subsequently at $\sim 1 \mathrm{D}(20 \mathrm{~cm})$ intervals up to $15.5 \mathrm{D}$

downstream of the pile centre. For the wave only experiments, measurements were taken from $0.75 \mathrm{D}$ to 4.5 D down-wave of the pile centre. Measurements were taken at locations around the perimeter of the pile at a distance of $\sim 0.75 \mathrm{D}$ from the pile centre. Measurements were also made with no pile in the basin, at the location of the pile, in order to quantify baseline conditions for mean flows, orbital velocities and turbulence.

\subsection{Data analysis}

Mean current values were calculated as the resultant velocity from the two time-averaged horizontal orthogonal components of the velocity data. This allowed for changes in flow direction in the horizontal plane around the pile.

$U_{c}=\sqrt{\left.\langle u\rangle^{2}+<v\right\rangle^{2}}$

where $v$ is the current in line with the main pumped current, $u$ is the current across the pumped current axis, and $<>$ denotes time averaging. 
Turbulent kinetic energy in the mean flow was calculated as

$k=0.5\left(<u^{\prime 2}>+<v^{\prime 2}>+<w^{\prime 2}>\right)$

where $u^{\prime}, v^{\prime}$ and $w^{\prime}$ denote the velocity fluctuations about the mean (following Nielsen et al. (2011) and Rogan et al. (2016)).

In the current only cases, the flow variability quantification associated with eddy shedding was considered analogous to orbital velocity calculation. Wave orbital velocity is often quantified as $u_{m}=$ $2 \sigma_{u}$, where $u_{m}$ represents the maximum orbital velocity, and $\sigma_{u}$ is the standard deviation of the velocity variance (e.g. Masselink et al., 2007). The magnitude of the flow variability was therefore parameterised using the standard deviation of the across axis flow velocity time series:

$u_{m}=2 \sqrt{\left\langle u^{\prime 2}\right\rangle}$

When waves are present, the orbital velocity orientation near the pile may deviate from the direction of wave advance. A resolved oscillatory current parameter $U_{w}$ was therefore calculated. For each horizontal axis, the orbital velocity $\left(u_{m}, v_{m}\right)$ was calculated. The resultant wave orbital velocity vector $U_{w}$ was then calculated, analogous to the mean current vector as:

$U_{w}=\sqrt{u_{m}^{2}+v_{m}^{2}}$

For current plus wave cases, the magnitude and direction of mean and oscillatory flows are influenced by the proximity to the pile, the relative contribution of currents and waves in each component direction, and the specific location around the pile. A velocity parameter $\left(U_{w c}\right)$ was calculated to represent the maximum possible velocity of a particle due to combined wave and currents:

$U_{w c}=\sqrt{\left(<u>+u_{m}\right)^{2}+\left(<v>+v_{m}\right)^{2}}$

Spectral analysis of the velocity time series was used to identify frequency of eddy shedding, and to identify the processes associated with waves and vortices separately. Spectral analysis was carried out using Matlab, following the approach of Oppenheim and Schafer (1975). Velocity spectra of the $u, v$ and $w$ time series were calculated using time series of length 16384 points (256 seconds), divided into 8 non-overlapping sections of length 2048 points, and 7 overlapping sections, with 50\% overlap. Sections were Hanning windowed and Fast Fourier Transformed to give 1024 energy estimates between 0 and the Nyquist frequency $(32 \mathrm{~Hz})$, with a frequency resolution of $9.7 \times 10^{-4} \mathrm{~Hz}$. The resulting spectra have 27.32 degrees of freedom. The $95 \%$ confidence intervals were calculated using the approach of Jenkins and Watts (1968).

\section{Results}

\subsection{Downstream recovery of current}

Velocity profiles from different distances downstream of the pile for the representative case $(25 \mathrm{~cm} / \mathrm{s})$ are shown in Fig. 6 . Close to the pile $\left(n_{D}=0.75\right)$, the velocity profile is approximately vertical. A narrow boundary layer forms over the bottom $2 \mathrm{~mm}$. With increasing distance from the pile, the velocity at the top of the profile increases, and by 10.5 to 11.5 pile diameters, the values are approximately equal to the 'no pile' $(\infty)$ profile. 


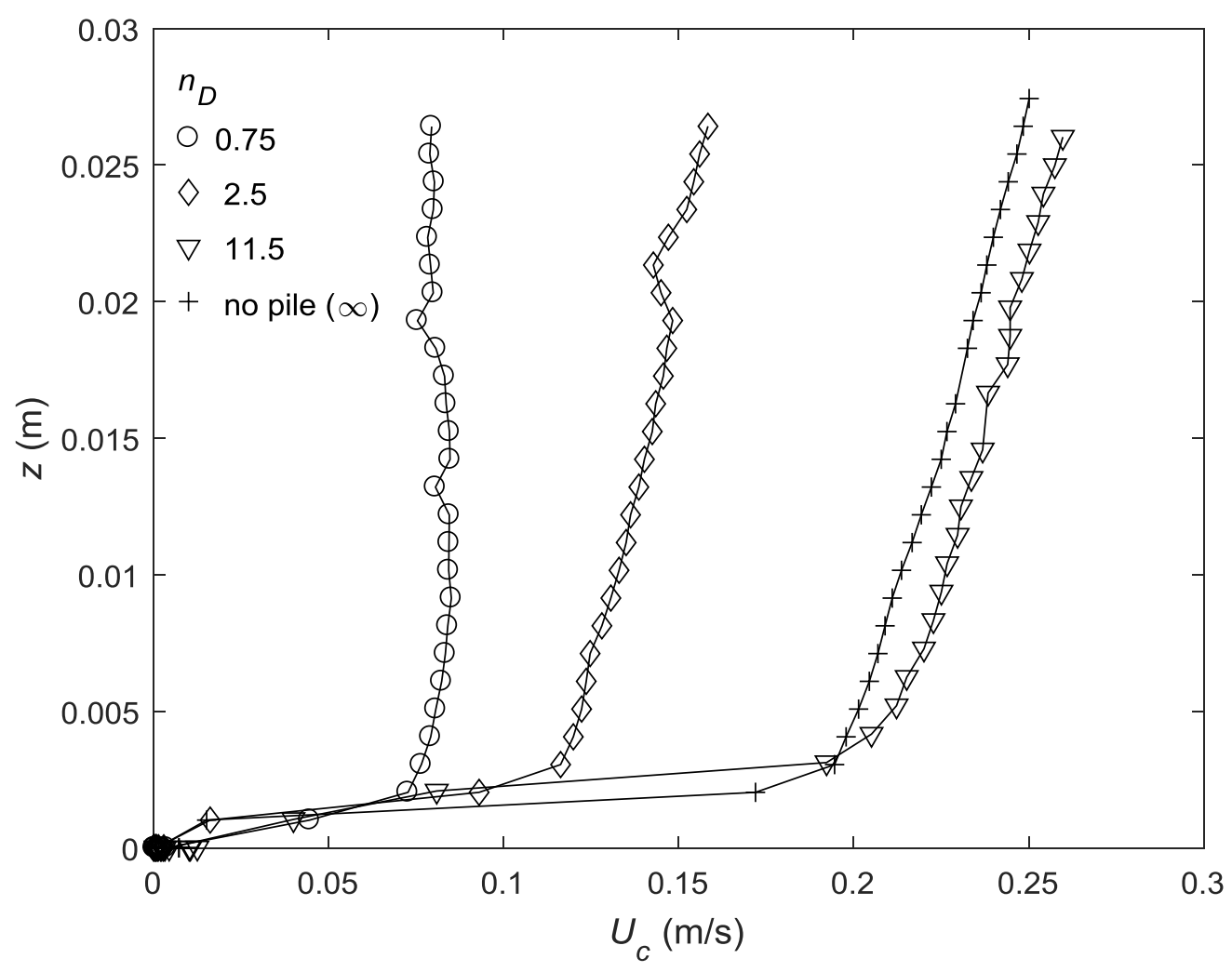

Fig. 6. Velocity profiles measured at different distances downstream of the pile, and with no pile. Distances are specified by the number of pile diameters downstream from the centre of the pile $\left(n_{D}\right)$.

By considering only the top of the measured profiles, it is possible to observe the trend in mean flow strength, and quantify the region affected by the pile (Fig. 7). Consistent with the profiles above, the velocity returned to full flow strength by $10.5 \mathrm{D}$ from the pile center. The lower horizontal line on Fig. 7 indicates 5\% less than the background flow. Using this line, it is possible to identify that the flow returned to within $5 \%$ of the background flow by 8.3 D. Fig 7 also shows the flow velocities calculated using the potential flow model (equations 1-3), with a background flow rate of $0.25 \mathrm{~m} / \mathrm{s}$. The potential flow model does a reasonable job of predicting the general form of the curve, but overpredicts the velocities, and therefore underestimates the downstream distance affected by the pile.

The recovery distance for the mean flow was investigated for different flow velocities. A similar trend arises for all current speeds tested (Fig. 8). To compare the downstream profiles, the velocity was expressed as a fraction of the measured current when there was no pile present. The general trend of the normalised flow evolution was similar across the range of flow speeds. The flow recovered to within 5\% of the background level (i.e. no-pile level) in a distance downstream between 6 and $10.6 \mathrm{D}$. For the 5 test cases, the average distance downstream required for flow recovery was $8.3 \mathrm{D}$.

The normalised velocities (from Fig 8) at each downstream location were averaged for each distance downstream (Fig 9a). The potential flow model clearly overestimates the flow velocity (Fig 9a). Assuming $U_{\infty}=U_{c-n p}$, the general form of a curve that follows the data more closely (Fig $9 \mathrm{~b}$ ) is given by a Michaelis-Henton model.

$\frac{U}{U_{\infty}}=\frac{\alpha n_{D}}{\beta+n_{D}}$ 
The data points at $n_{D}=1.5$ and $n_{D}=5.5$ fall below the line that the rest of the data appear to follow. Omitting these points and performing a regression analysis of the Lineweaver-Burk linearised form of the equation (Kassab, 1989) gives $\alpha=1.2179$ and $\beta=2.3303$ with an $\mathrm{R}^{2}$ of 0.998 . It is unclear why these two points do not lie on the line, but previously unidentified issues with pump speed variability are possible. The curve indicates that the velocity returns to within $5 \%$ of the background value by $n_{D}$ $=8.26$.

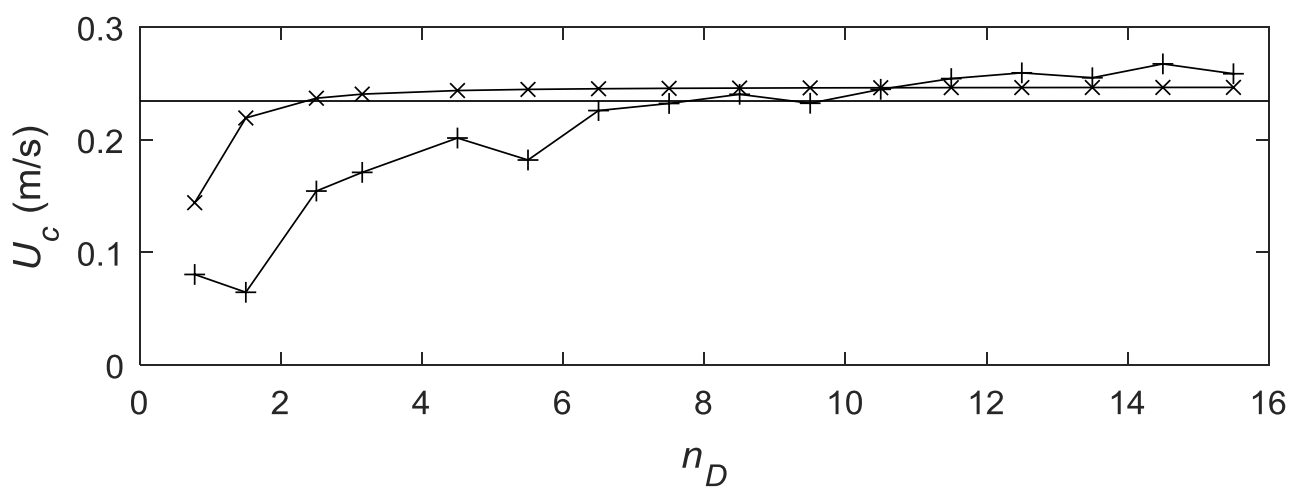

Fig.7. Velocity plotted against number of pile diameters downstream of the centre of the pile. The pile centre is at $n_{D}=0$. Variation in mean flow velocity $U_{c}(+)$ as measured at $z=0.025 \mathrm{~m}$ above the bed, and modelled using potential flow theory (x). The horizontal line is $5 \%$ less than the background flow rate.

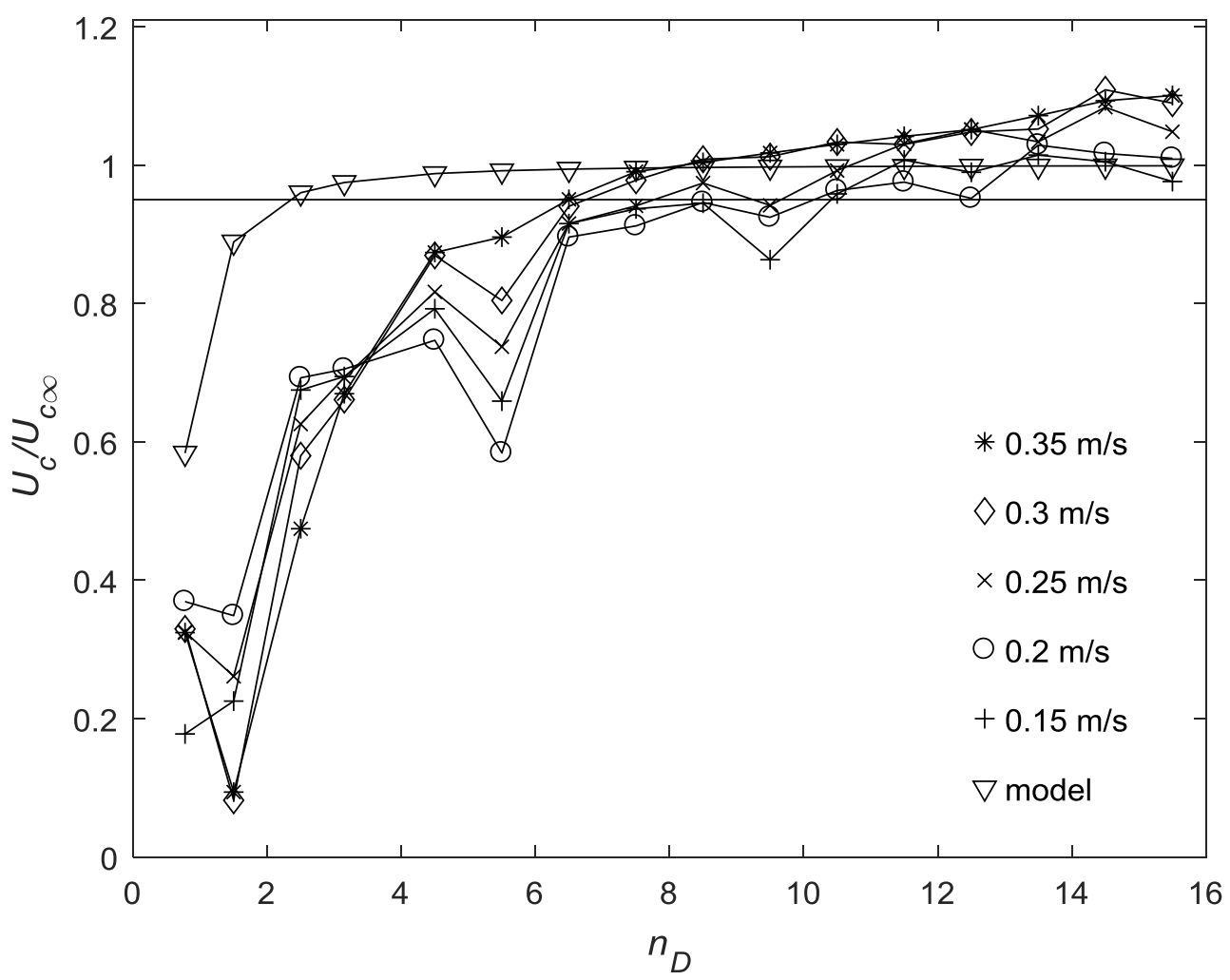

Fig. 8. Variation in velocity downstream of the pile for the 5 different flow speeds tested, and for the potential flow model. Velocity is expressed as a fraction of the no-pile velocity. $n_{D}$ specifies the number of pile diameters downsream of the pile centre from which the measurement was taken. Horizontal lines indicate background flow and 95\% of background flow. 

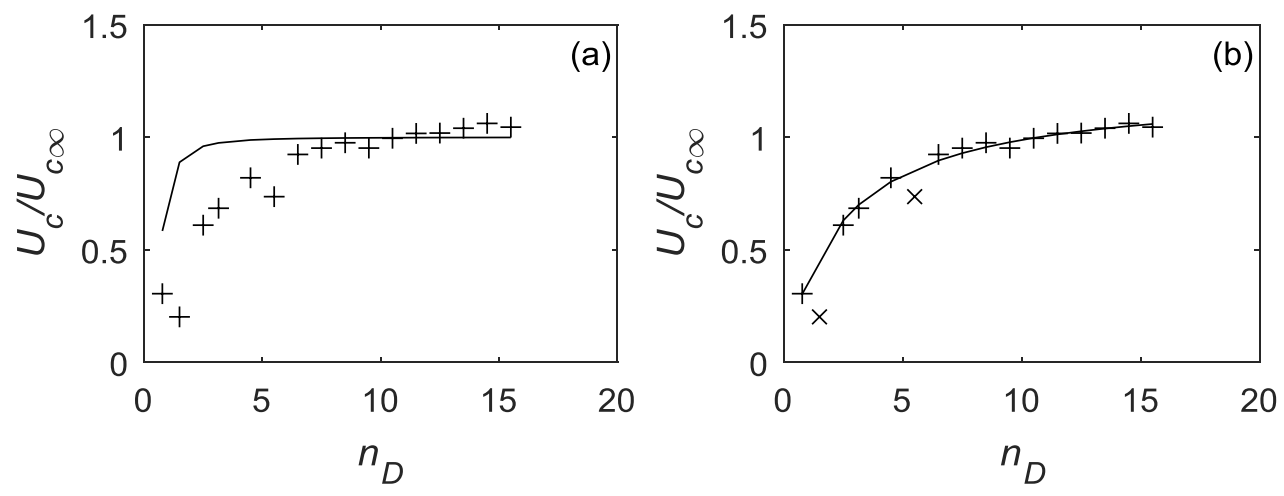

Fig. 9. (a) The measured average normalised velocity at each downstream location (+), compared to the potential flow model. (b) shows an empirical parametric model of the data points, excluding data points marked (x).

The recovery distance showed a weak relationship with the background flow velocity (Fig. 10). Linear regression suggested that as the the background velocity increased, the recovery distance reduced slightly. In the range $0.15 \mathrm{~m} / \mathrm{s}$ to $0.35 \mathrm{~m} / \mathrm{s}$, the recovery distance in terms of the number of pile diameters for the basin tests was given by

$n_{D}=14.1-22.9 U_{c \infty}$

with an $\mathrm{r}^{2}$ of 0.9 . The actual distance downstream that is affected $x$ is given by $x=n_{D} D$. In prototype, a pile diameter of $5 \mathrm{~m}$ and recovery distances of up to 10 pile diameters suggest an affected distance of up to $50 \mathrm{~m}$. With the laboratory values scaled to prototype conditions in the range 0.75 to $1.75 \mathrm{~m} / \mathrm{s}$, the equation specifying the distance downstream in the field that it takes for the flow to return to within $5 \%$ of the backgroud value is therefore:

$x=\left(14.1-4.58 U_{c \infty}\right) D$

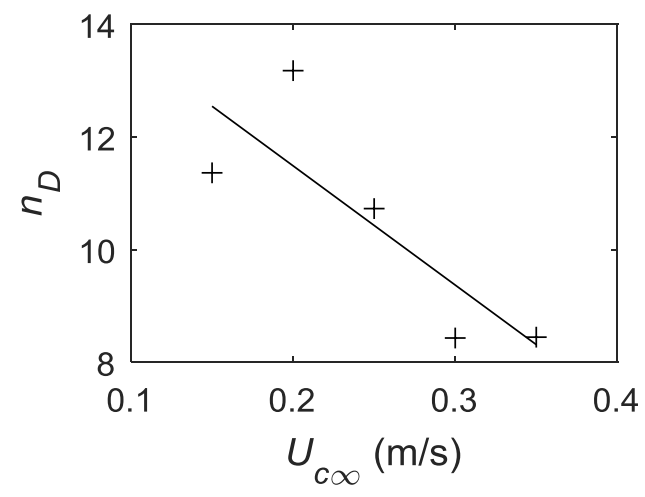

Fig. 10. Recovery distance was identified from the laboratory data by the number of pile diameters downstream $\left(n_{D}\right)$ it takes to get to within $5 \%$ of the no-pile velocity $\left(U_{c \infty}\right)$. 


\subsection{Turbulence}

A section of data illustrating the variability in the across stream flow from $2.5 \mathrm{D}$ downstream of the pile in $0.25 \mathrm{~m} / \mathrm{s}$ flow is shown in Fig 11. Turbulent oscillations with a timescale of several seconds are clear. For this flow strength, analysis using the Strouhal number indicates vortex shedding at a frequency of $0.237 \mathrm{~Hz}$. This corresponds to a period of 4.2 seconds. This period is shown as a horizontal bar on the time series in Fig. 11.

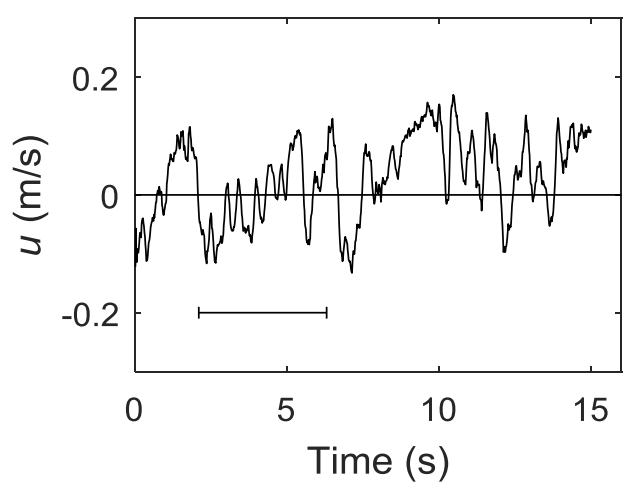

Fig. 11. Oscillations in the cross axis flow $(u)$, downstream of the pile, indicative of turbulence. The horizontal bar indicates the period predicted by the Strouhal analysis ( 4.2 seconds in this case). The mean flow strength is $0.25 \mathrm{~m} / \mathrm{s}$ (orthogonal to this data).

In the data in Fig. 11 it is evident that there is some variability in the exact duration between flow peaks. The data were analysed using spectral analysis to identify a quantified oscillation frequency. Spectra of the three axes of the flow are shown in Fig. 12. The frequency indicated by the Strouhal number is shown as the vertical line on the spectrum. Most energy is contained in the across axis flow $(u$, red), and this peaks at the Strouhal frequency. The along axis flow (v, black) has slightly less energy, but also suggests a peak near the Strouhal frequency. There is less energy present in the vertical flow variance ( $w$, green), suggesting that the majority of the variability arises from horizontal eddies associated with vortex shedding.

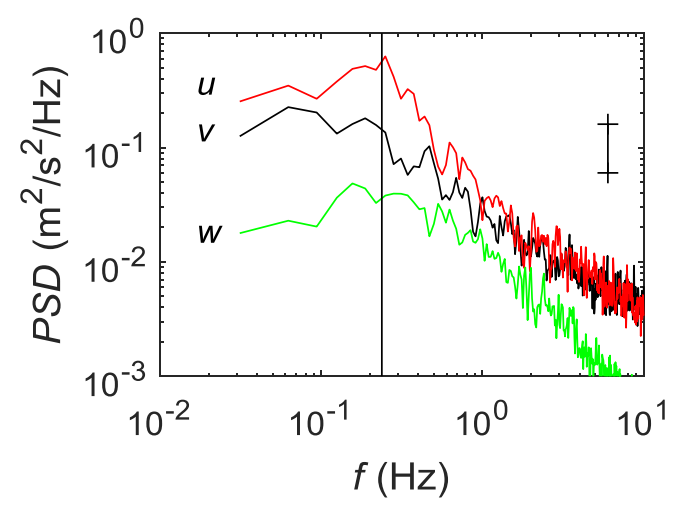

Fig. 12. Velocity spectra (Power Spectral Density, $P S D$ ) of currents across the main flow axis $(u$, red), in line with the flow ( $v$, black) and in the vertical direction ( $w$, green). The vertical line indicates the frequency indicated by the Strouhal number $(0.237 \mathrm{~Hz})$. The whisker bar indicates the $95 \%$ confidence limits. This data is from $2.5 \mathrm{D}$ downstream of the pile centre. 


\subsection{Downstream variation in turbulence}

The distribution of turbulence downstream of the pile, as parameterised by $k$, is shown in Fig. 13. The data indicate a peak in turbulence at $1.5 \mathrm{D}$ (i.e. $1 \mathrm{D}$ downstream of the pile edge), followed by a reduction with distance downstream. The turbulence does not get back to within 5\% of the background level within the distance measured $(15.5 \mathrm{D})$. The initial reduction in turbulence at $0.75 \mathrm{D}$ was not evident in the data of Rogan et al. (2016).

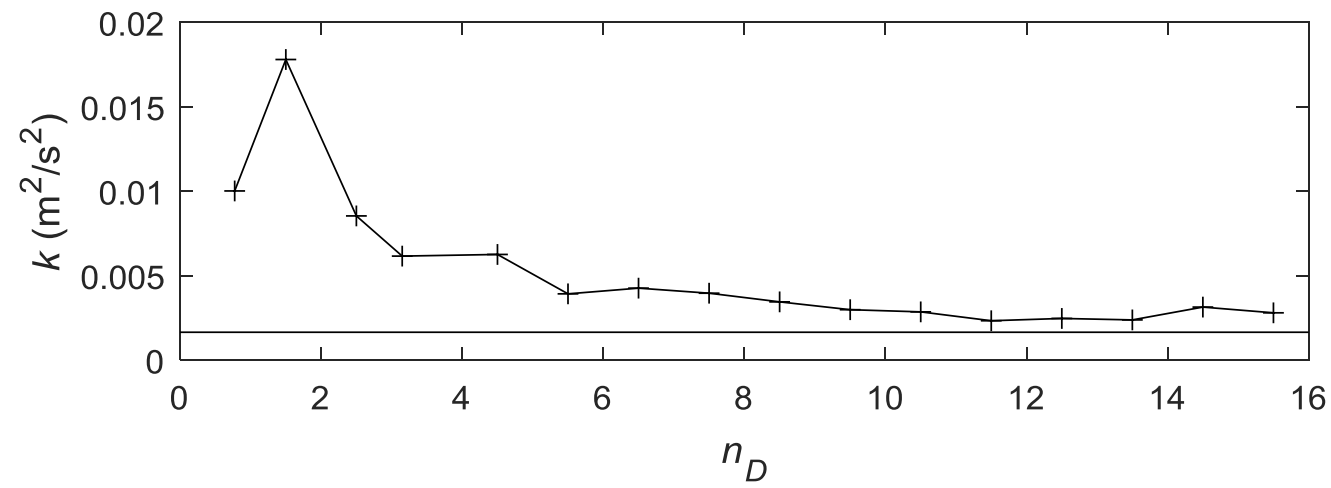

Fig. 13. Turbulence plotted against number of pile diameters downstream of the centre of the pile $\left(n_{D}\right)$. The pile centre is at $n_{D}=0$. The horizontal line identifies $k$ with no pile $(0.25 \mathrm{~m} / \mathrm{s})$.

Data from the other flow velocities tested indicate a very similar structure to the turbulence (Fig. 14). The general level of turbulence increases with flow velocity, and the magnitude of the peak at $1.5 \mathrm{D}$ increased with flow velocity. The position of the peak in the plot remains at $1.5 \mathrm{D}$, however it is noted that the resolution of the data is coarse. Further experiments with finer resolution would be useful to determine the position of the peak.

In order to parameterise the variability in turbulence, Rogan et al. (2016) normalised $k$ using the mean background velocity squared. The application of this approach collapses the peak and near pile turbulence values to approximately the same curve (Fig. 15).

For this data, the non-dimensional turbulence values at each distance downstream were then averaged. A curve of the form:

$\frac{k}{U_{c \infty}^{2}}=\alpha+\frac{\beta}{n_{D}}$

fitted the data (Fig 16), with constants $\alpha=0.0119$ and $\beta=0.3366$, and an $\mathrm{R}^{2}$ of 0.97 . This curve applies from the peak at 1.5 $\mathrm{D}$ from the centre to the measured 15.5 D downstream.

The relative contributions to the total turbulence $(k)$ in the $u, v$, and $w$ directions were given by Nielsen et al. (2011), following Nezu and Nakagawa (1993), as 0.55, 0.28 and 0.17 respectively. In this data, the background (no pile) values were $0.56,0.36$ and 0.08 respectively. Fig. 17 shows the distribution of turbulence contributions downstream of the pile. The relative proportions of $u, v$ and $w$ turbulence remain approximately constant with distance downstream. The average contributions are $0.48,0.46$ and 0.06 to $u, v$ and $w$ respectively. There is however, a notable enhancement in the contribution of the across axis flow downstream of the pile compared to the expected background level, and the contribution of $k_{l} / k$ at $\mathrm{n}_{\mathrm{D}}=1.5$ reaches 0.59 . 


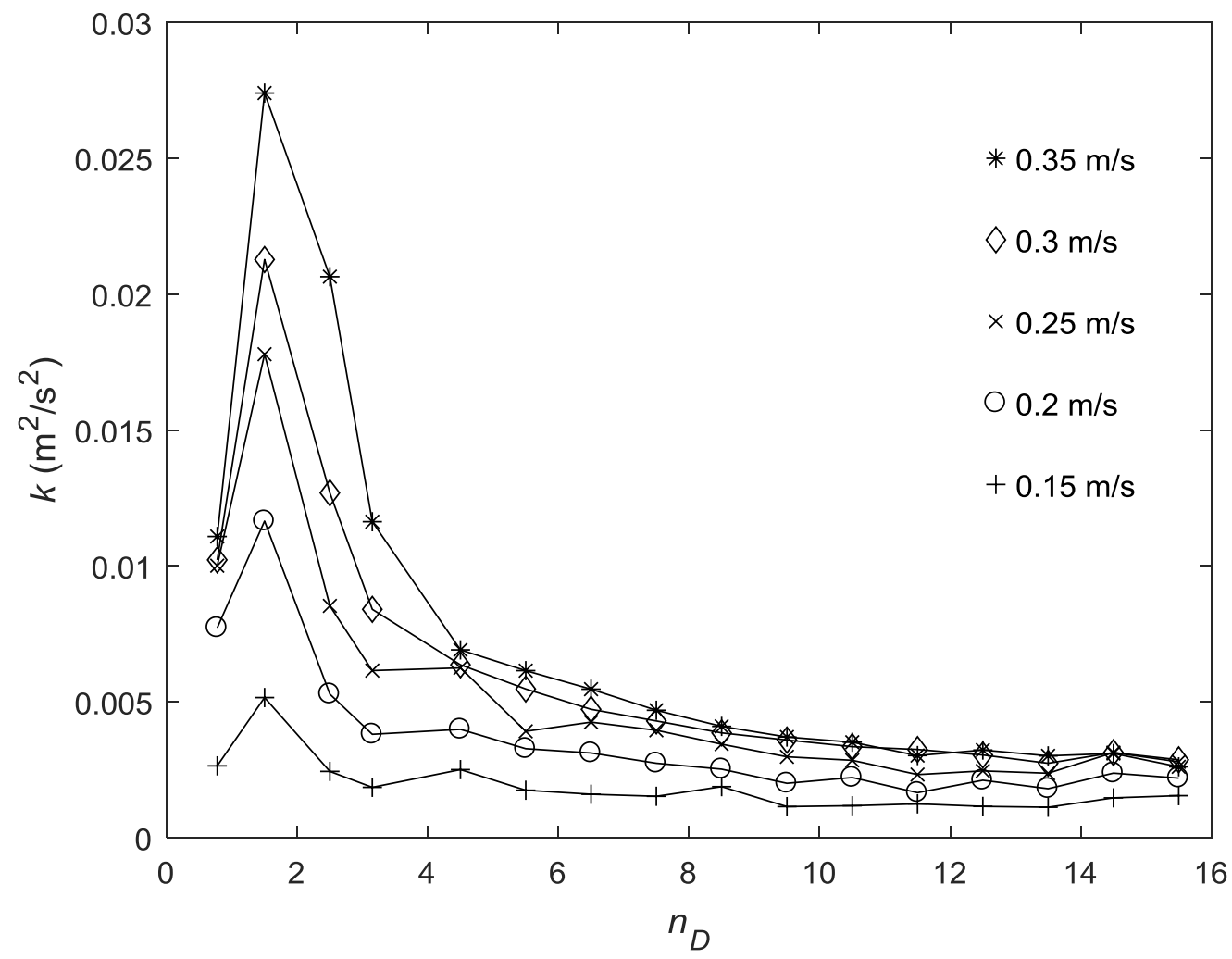

Fig. 14. Turbulence downsream of the pile at different flow speeds.

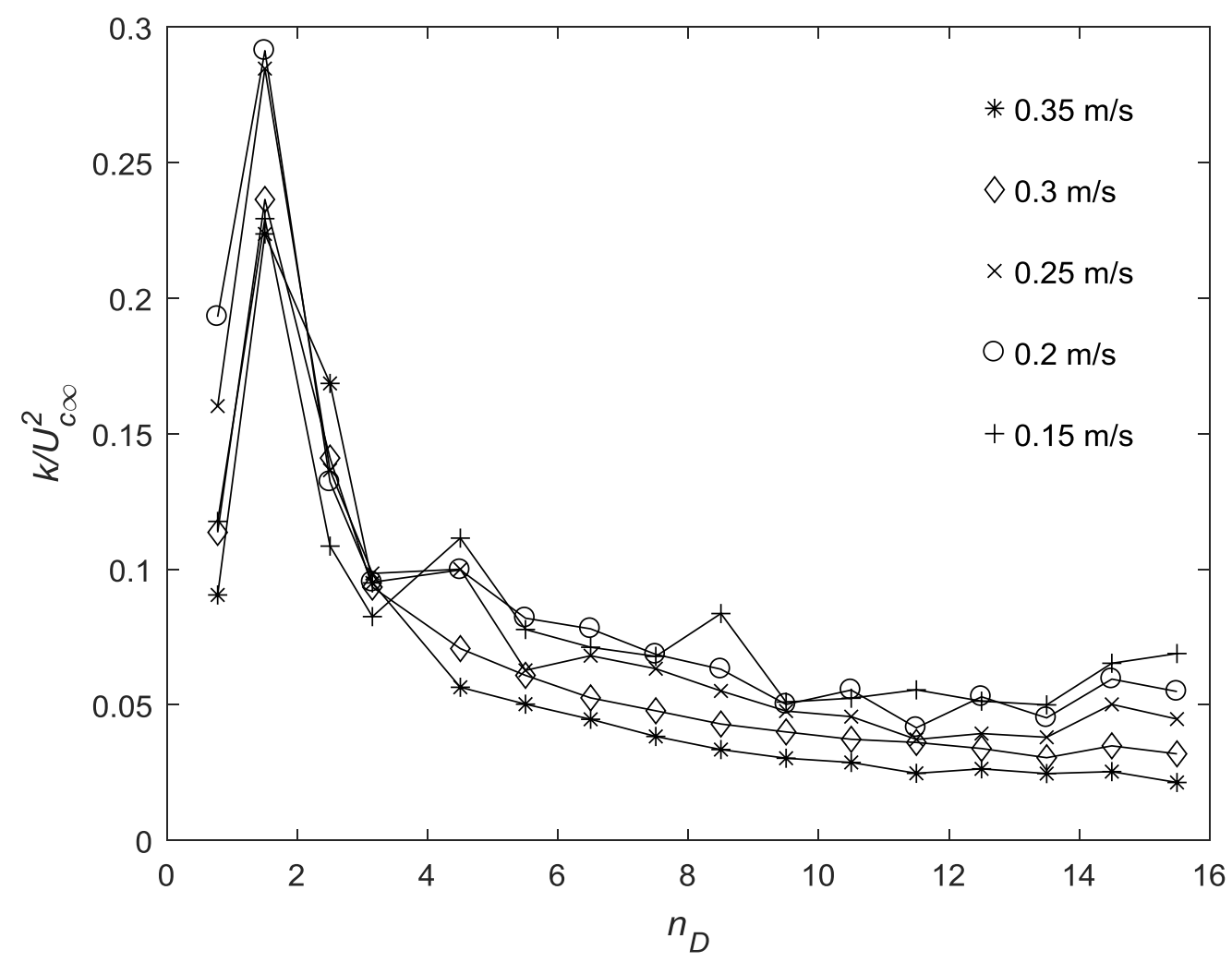

Fig. 15. Turbulence downsream of the pile at different flow speeds, normalised by no-pile velocity squared. 


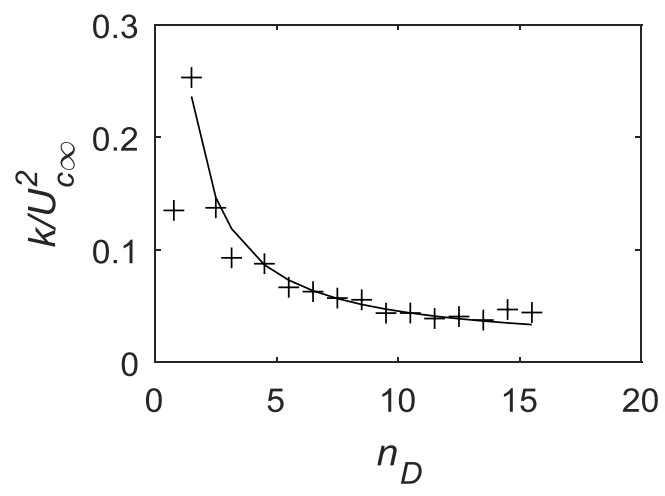

Fig. 16. Averaged normalized turbulence downsream of the pile at different flow speeds, parameterised with a reciprocal model for $n_{D}>1.5$.
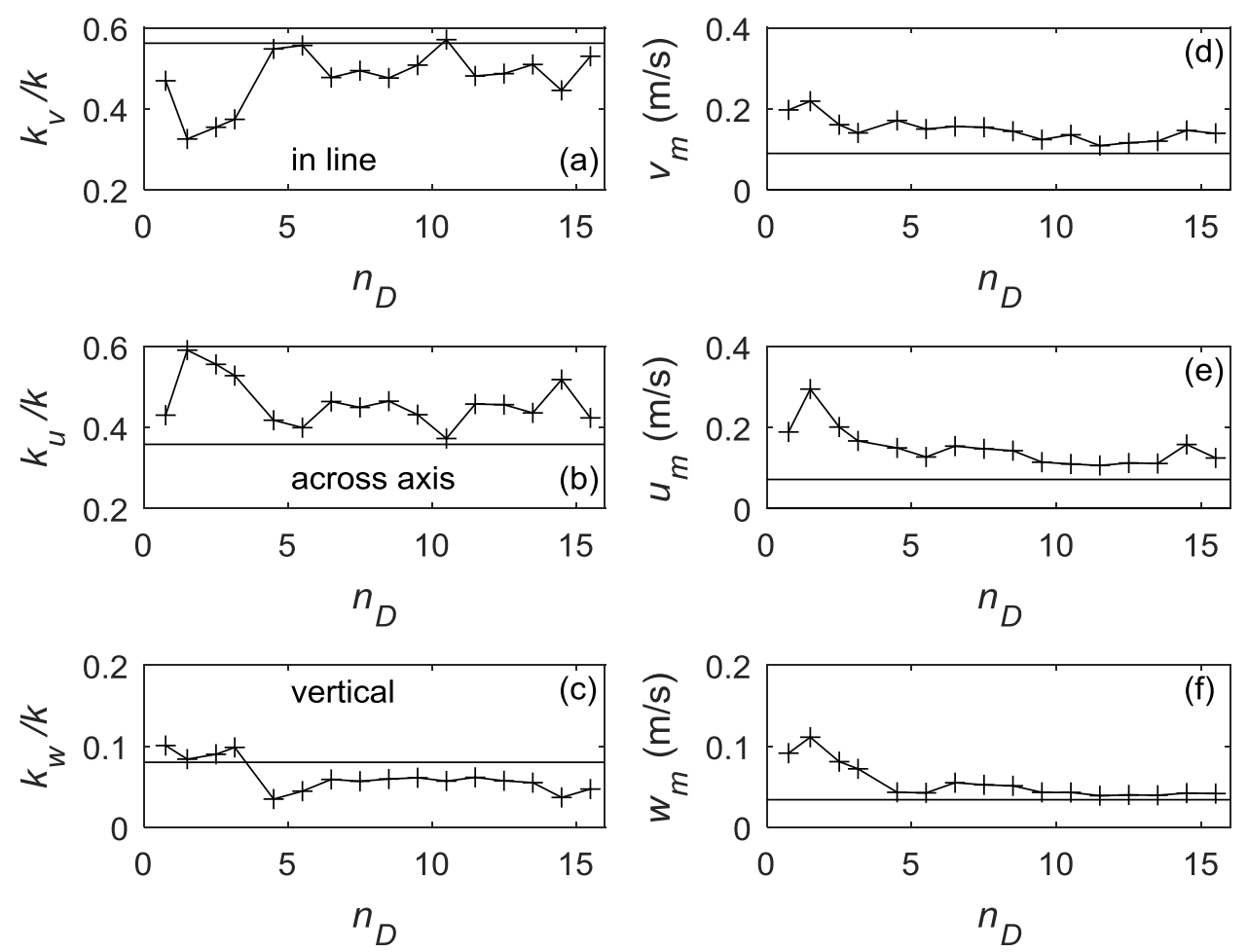

Fig 17. (a-c): Fractional contribution of components of turbulence to the total. $v$ and $u$ indicate flow variance in-line with, and across the free stream flow respectively. w identifies vertical flow. The horizontal line indicates the no pile case. (d-f): magnitude of the velocity indicated by the turbulence. Background flow is $0.25 \mathrm{~m} / \mathrm{s}$ in the $v$ direction, and the horizontal line indicates the background level of turbulence in the no-pile case.

The magnitude of the flow variability (equation 10) are quantified in Fig 17 for the $0.25 \mathrm{~m} / \mathrm{s}$ representative case. A key point of interest is that the magnitude of the horizontal components of the turbulent oscillations near the pile are close to the magnitude of the mean flow $(0.25 \mathrm{~m} / \mathrm{s})$. This extends to the magnitude of the across-axis flow, which peaks at $0.3 \mathrm{~m} / \mathrm{s}$, at $1.5 \mathrm{D}$ downstream of the pile centre. The general trend downstream follows the general shape of the turbulence decay. The horizontal components do not quite reduce to the background level within the distance measured. 


\subsection{Flow strengths around the pile}

Flow measurements were made at different compass points around the pile, at a distance of $\sim 0.75 \mathrm{D}$ away from the centre of the pile (Fig. 18). Measurements upstream of the pile $\left(0^{0}\right)$ were approximately logarithmic in profile, and similar in profile to those indicated by Roulund et al. (2005). They are however, reduced in magnitude considerably from the 'no-pile' case. Flow strengths increase around the pile, and are maximum at the side of the pile $\left(90^{\circ}\right)$. Immediately downstream of the pile $\left(180^{\circ}\right)$, the flow strengths are reduced (as described above). At $135^{\circ}$, there is evidence that in the lower part of the water column the flow strengths remain higher, possibly indicating that flow separation occurs further around the pile in the lower part of the water column than in the upper part.

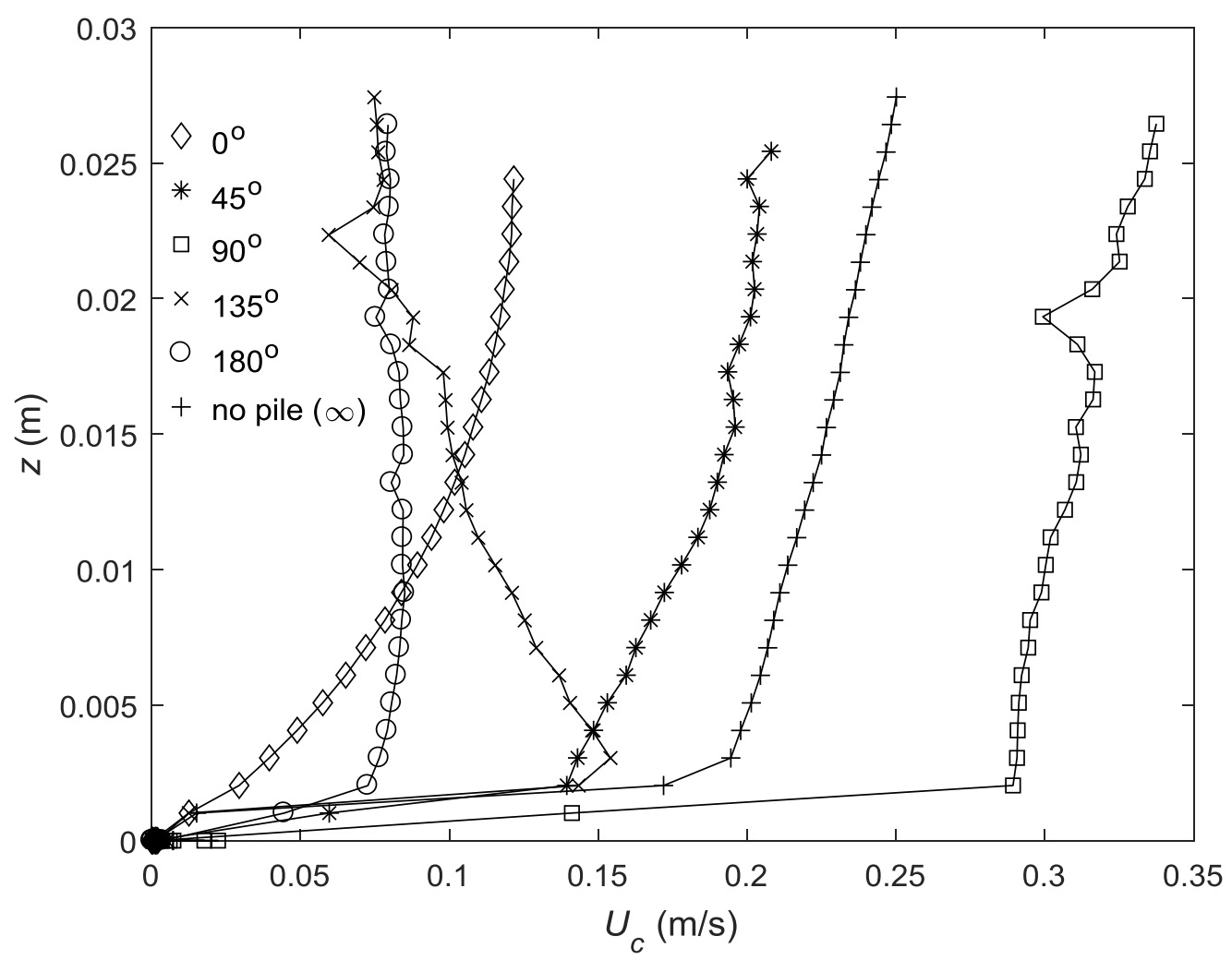

Fig. 18. Velocity profiles mapped around the pile. Measurements are taken $0.75 \mathrm{D}$ from the centre of the pile. $0^{\circ}$ is upstream of the pile, $90^{\circ}$ is to the side, $180^{\circ}$ downstream.

For the purpose of designing cabling, a velocity value is required for the mean flow strength near the pile, from obtainable predictions of tidal current speeds. Normalising the measured velocity against the 'no-pile' velocity quantifies the flow enhancement / reduction. The data shows that the flow magnitude at the side of the pile was 1.35 times the background (no-pile) flow rate (Fig. 19). Flow magnitudes were reduced to 0.49 and 0.31 times the background flow rate at the front and back of the pile respectively.

The potential flow model (equations 1-3) gave a reasonable prediction of the flow modification in front of the pile and to the side, however it over-predicted the flow in the 'stern quarter' at 135 to 180 degrees (Fig. 19). Levels of turbulence were calculated for the different positions around the pile (Fig 20). A significant increase in turbulence occurred at $135^{\circ}$ (the stern quarter of the pile) and was still evident at $180^{\circ}$ (the downstream side). This increase in turbulence coincided with the velocity reduction at these points, when compared to the potential flow model (Fig 19). 


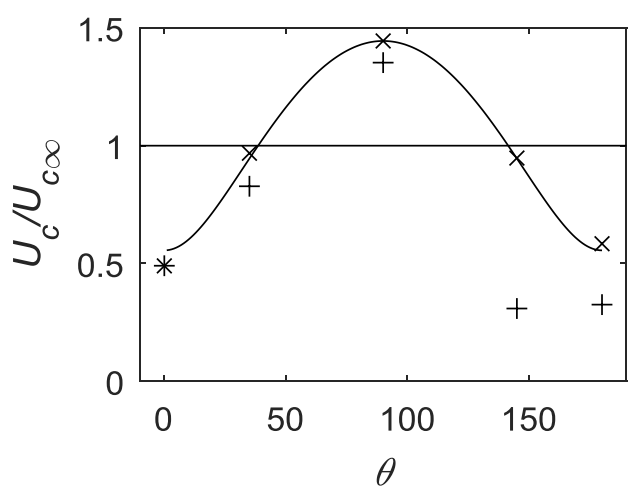

Fig. 19. Velocity data, normalised by the no pile velocity, mapped around the pile $(+) .0^{\circ}$ is upstream, $180^{\circ}$ is downstream. The horizontal line is the no-pile case. The curve shows the potential flow model velocity distribution at $0.75 \mathrm{D}$ from the pile centre. The $\mathrm{x}$ values mark the potential flow model values calculated exactly at the sensor positions (close to $0.75 \mathrm{D}$ ).

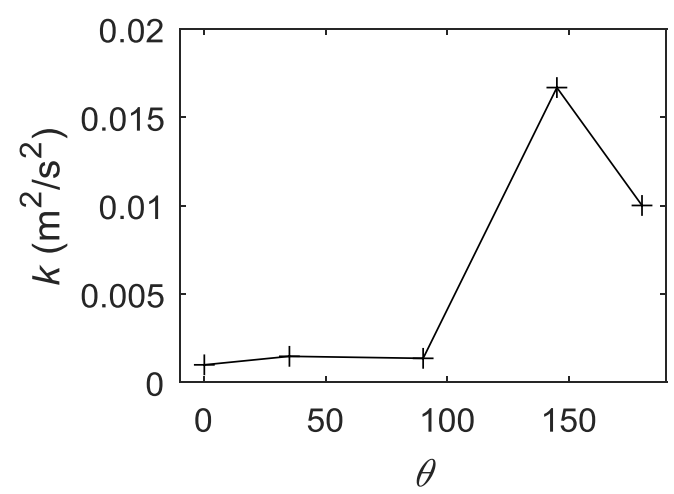

Fig. 20. Turbulence $(k)$ calculated at different positions around the pile, at $\sim 0.75 \mathrm{D}$ from the pile centre. $0^{\circ}$ is upstream.

The proportion of the increase at the side of the pile (i.e. 'flow enhancement') was investigated for different flow strengths. Fig. 21 shows a relatively constant flow enhancement in the range 0.15 to $0.35 \mathrm{~m} / \mathrm{s}$. The average enhancement is 1.36 of the background flow. The potential flow model slightly exaggerates the flow enhancement, and indicates a value of 1.44 times the background flow at $0.75 \mathrm{D}$ from the pile centre.

\subsection{Waves only}

Wave orbital velocities were calculated at various distances down-wave of the monopile in the representative windsea case. The vertical profile of the orbital velocity maxima are shown in Fig. 22. The general vertical form of the profiles is similar, regardless of distance from the pile, with a relatively narrow boundary layer, and approximately vertically homogeneous velocity profiles.

The near-pile data $(0.75 \mathrm{D})$ shows a reduced resolved oscillatory velocity, compared to values further from the pile. Data from $\mathrm{z}=0.025 \mathrm{~m}$ were analysed to illustrate the variation in the wave orbital velocity with distance away from the pile (Fig 23a). The near pile velocity is 0.64 times the no pile test case. The data indicates that between $0.75 \mathrm{D}$ and $3.4 \mathrm{D}$ the resolved wave orbital velocity has 
returned to background level. It is slightly unclear if the return occurs by $1.65 \mathrm{D}$, or just after $2.4 \mathrm{D}$. The orbital velocity appears to increase slightly beyond the no pile case at 3.4 and 4.4 D. Despite the minor ambiguity, it appears that the reduction in orbital velocity for waves is much more localised than for currents.

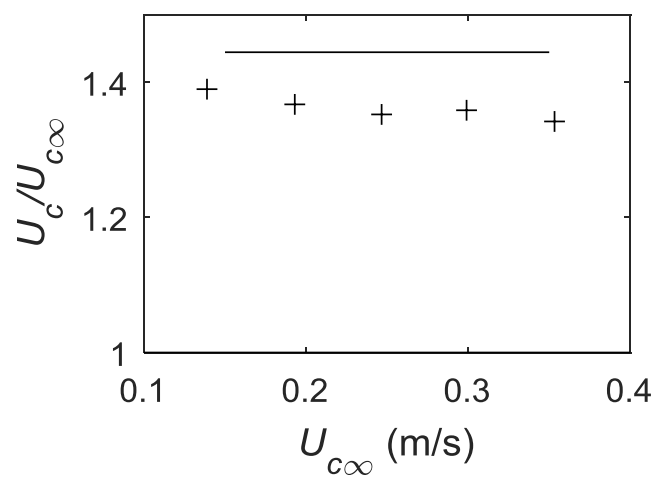

Fig. 21. Flow enhancement at the side of the pile $\left(90^{\circ}\right)$ for different background flow speeds. The horizontal line indicates the enhancement indicated by the potential flow model.

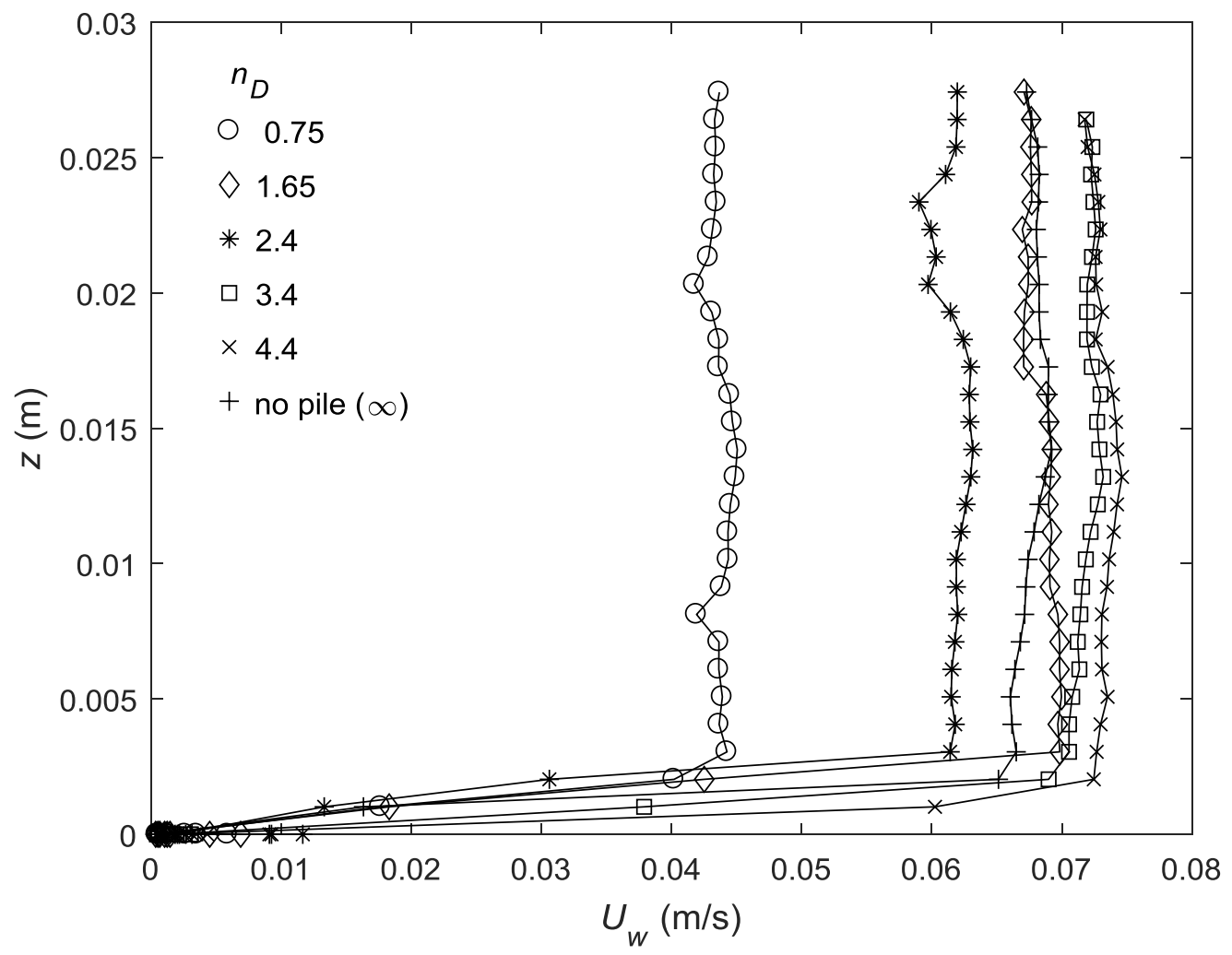

Fig. 22. Vertical profiles of resolved orbital velocity $\left(U_{w}\right)$ shown at different distances from the pile.

The orbital velocities downstream of the pile $\left(U_{w}\right)$ were normalised by the no-pile case $\left(U_{w \infty}\right)$ and were compared to the distribution suggested by the steady flow potential flow model. Fig $23 \mathrm{~b}$ suggests that the potential flow model does a reasonable job of predicting the general form of the wave orbital velocity maxima in this case. 

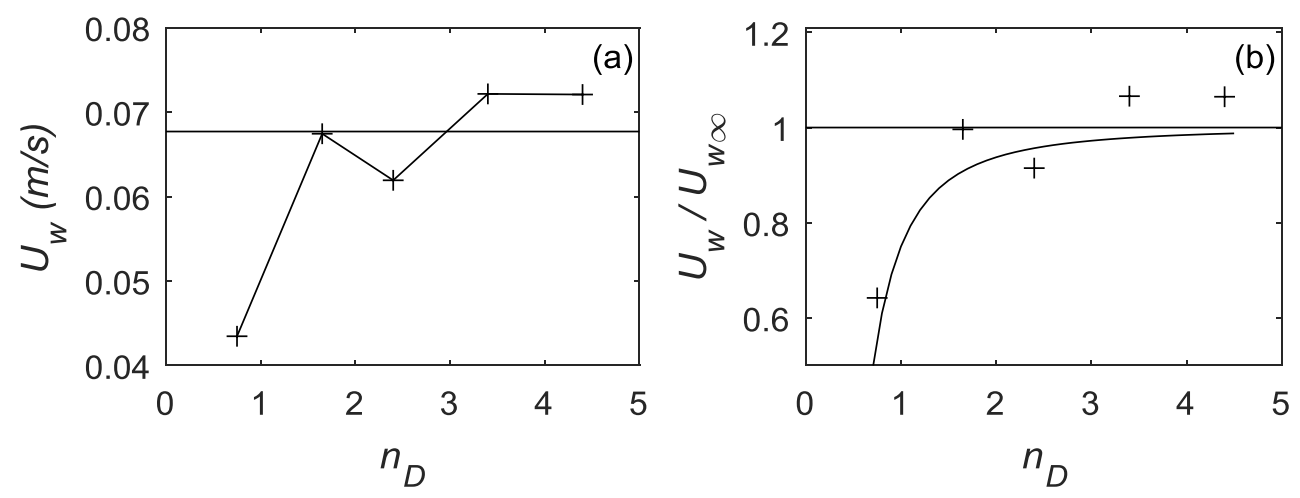

Fig. 23. (a) Orbital velocity maximum $\left(U_{w}\right)$ variation with distance downstream, expressed as number of pile diameters downstream of the pile centre $\left(n_{D}\right)$ for the windsea case. (b) The orbital velocity maximum was normalised using the no-pile case $\left(U_{w \infty}\right)(+)$, and plotted with model predictions using the potential flow model (solid line).

The distribution of wave orbital velocity profiles around the pile is shown in Fig. 24. Orbital velocities are reduced immediately in front (up-wave) of the pile $\left(0^{\circ}\right)$ and behind it (down-wave, $\left.180^{\circ}\right)$. Orbital velocities are maximum to the side $\left(90^{\circ}\right)$, and are enhanced at both $45^{\circ}$ (towards front) and $135^{\circ}$ (towards back) compared to the no-pile case. At $90^{\circ}$ and $45^{\circ}$ there is a small indication of increased flow near the bed, however the general form of the profiles is that they are approximately vertically homogenous. This is consistent with the observation by Sumer et al. (1997) that no horseshoe vortices form at low $\mathrm{KC}$ number.

The general distribution of wave driven flows around the pile for three different wave cases (windsea, swell, storm) are shown in Fig. 25. In all three cases, flows were reduced below background level both up-wave $\left(0^{\circ}\right)$ and down-wave $\left(180^{\circ}\right)$ of the pile. In all three cases, maximum orbital velocity enhancement was at the sides of the pile $\left(90^{\circ}\right)$.

For the windsea representative test case identified here, the orbital velocities at the side of the pile $\left(90^{\circ}\right)$ were up to 1.66 times larger than the background values. In swell conditions the orbital velocity enhancement was up to 1.85 times the background orbital velocity $\left(\right.$ at $90^{\circ}$ ). In storm conditions, the magnitude of the orbital velocity increased significantly (as would be predicted for no-pile), but the enhancement by the pile was slightly less than windsea conditions, at 1.53 times the no-pile case.

Fig. $25 \mathrm{~b}$ also shows the curve predicted using the potential flow model at $0.75 \mathrm{D}$ (solid line) and at the exact sensor positions. The model follows the general form of the enhancement well. The enhancement is slightly underestimated by the model, which suggests an increase of 1.51 times the background at $0.7 \mathrm{D}$ and $90^{\circ}$ (compared to the measured increase of 1.53 to 1.85 at this point).

\subsection{Currents and Waves}

Data from tests on orthogonal waves and currents are shown in Fig. 26. The plot shows data for the representative case test with a mean flow of $0.25 \mathrm{~m} / \mathrm{s}$ and windsea wave conditions. Velocities were calculated from the combination of currents and waves $\left(U_{w c}\right)$, incorporating both $u$ and $v$ velocities, calculated using equation 12 . The orientation of the polar co-ordinate system follows the currents data, with $0^{\circ}$ as up-current, angles increasing clockwise, and $270^{\circ}$ as up-wave. 


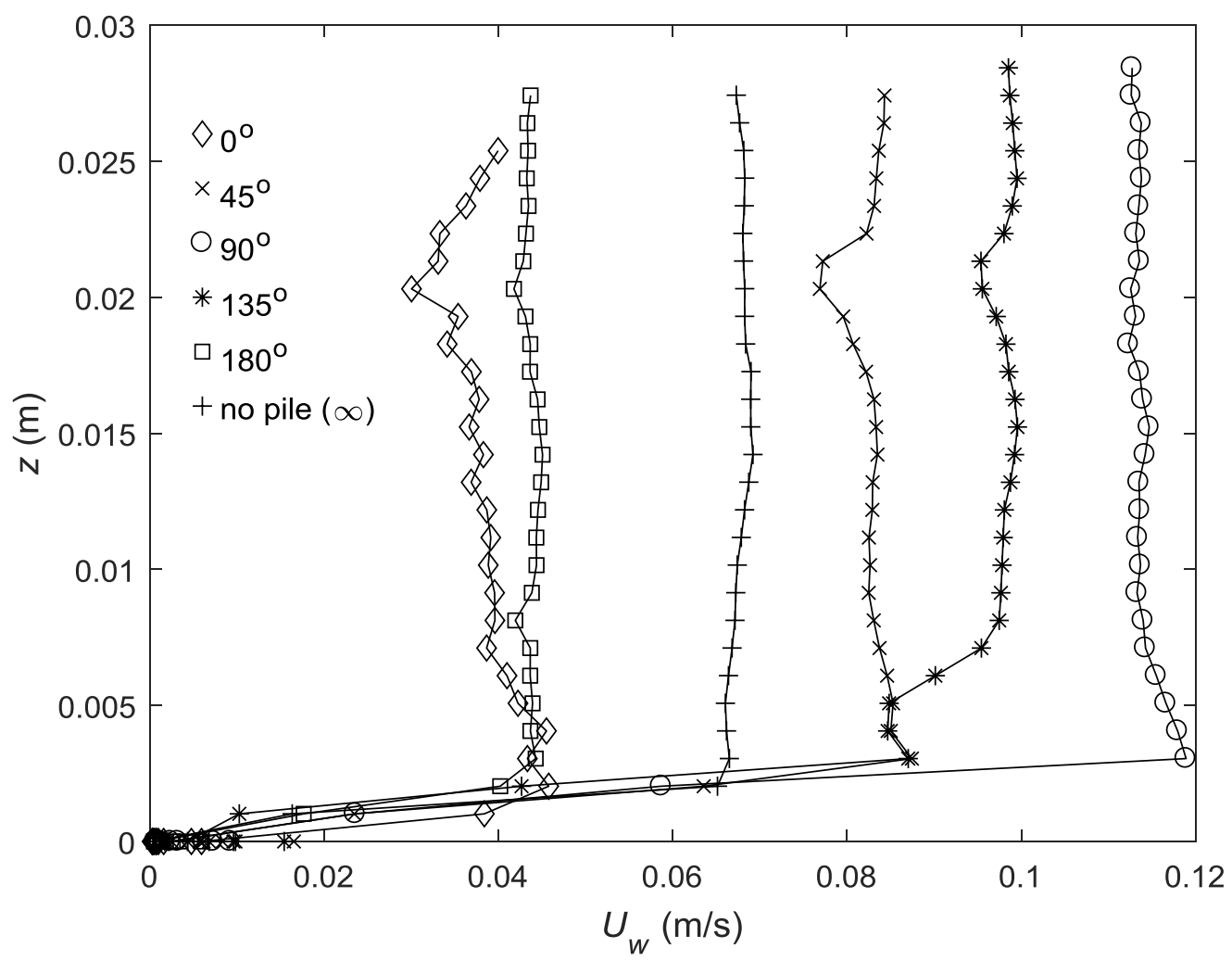

Fig. 24. Near bed orbital velocity maximum $\left(U_{w}\right)$ profiles at different positions around a pile, in modelled windsea conditions and with no pile. $0^{\circ}$ is 'up-wave', $90^{\circ}$ is to the side, and $180^{\circ}$ is down-wave.

Velocities were enhanced from the no-pile case at all points other than up-current and down-current. Velocities were maximum at the sides of the pile $\left(90-135^{\circ}\right.$ and $\left.270^{\circ}\right)$. Maximum near bed velocities were at $135^{\circ}$ and $225^{\circ}$, just downstream of the sides of the pile when viewed as a current type problem. A 'slice' through the plot at $z=0.025 \mathrm{~m}$ illustrates the general distribution of velocities (Fig. $27 \mathrm{a})$. Flows were least at the up-current position $\left(0^{\circ}\right)$, and largest at the current-sides $\left(90^{\circ}\right.$ and $\left.270^{\circ}\right)$. At the down-current $\left(180^{\circ}\right)$ position, flows did not return to the low flows measured at $0^{\circ}$. This is possibly due to horizontal mixing in the turbulent wake, assisted by the orthogonal waves. The flow enhancement of $U_{\mathrm{wc}}$ compared to the velocity with no pile $\left(U_{w c-\infty}\right)$ reaches 1.2 , and is maximum at the current-side of the pile (Fig. 27b).

Spectral analysis was carried out on velocity data immediately down current from the pile $(0.775 \mathrm{D}$ from the centre) in the current with orthogonal waves case (Fig. 28). The across-current flow velocity (u) shows peaks at both the wave frequency $(0.85 \mathrm{~Hz})$ and at $0.28 \mathrm{~Hz}$, close to the frequency of eddy shedding indicated by the Strouhal number $(0.237 \mathrm{~Hz})$ for this mean flow velocity $(0.25 \mathrm{~m} / \mathrm{s})$.

\section{Discussion}

Satellite imagery taken of suspended particulate matter suggests that the pile has the potential to impact water quality $\sim \mathrm{km}$ downstream (Vanhellemont and Ruddick, 2014). These visible effects require a point source of turbidity in the flow, rather than a modified velocity field, but do suggest that individual piles can have far reaching effects. Numerical models indicate that there may be small differences in velocity between the wake and the background flow over extensive distances (Rivier et al., 2014), however these differences will be difficult to detect in field or laboratory data over larger differences. The exact size of the wake will clearly depend on the lateral mixing that is present (either 
in nature or in the models). The experiments here suggest that a rule of thumb for engineering purposes is that the downstream effects have a length scale of approximately 8 to $10 \mathrm{D}$.
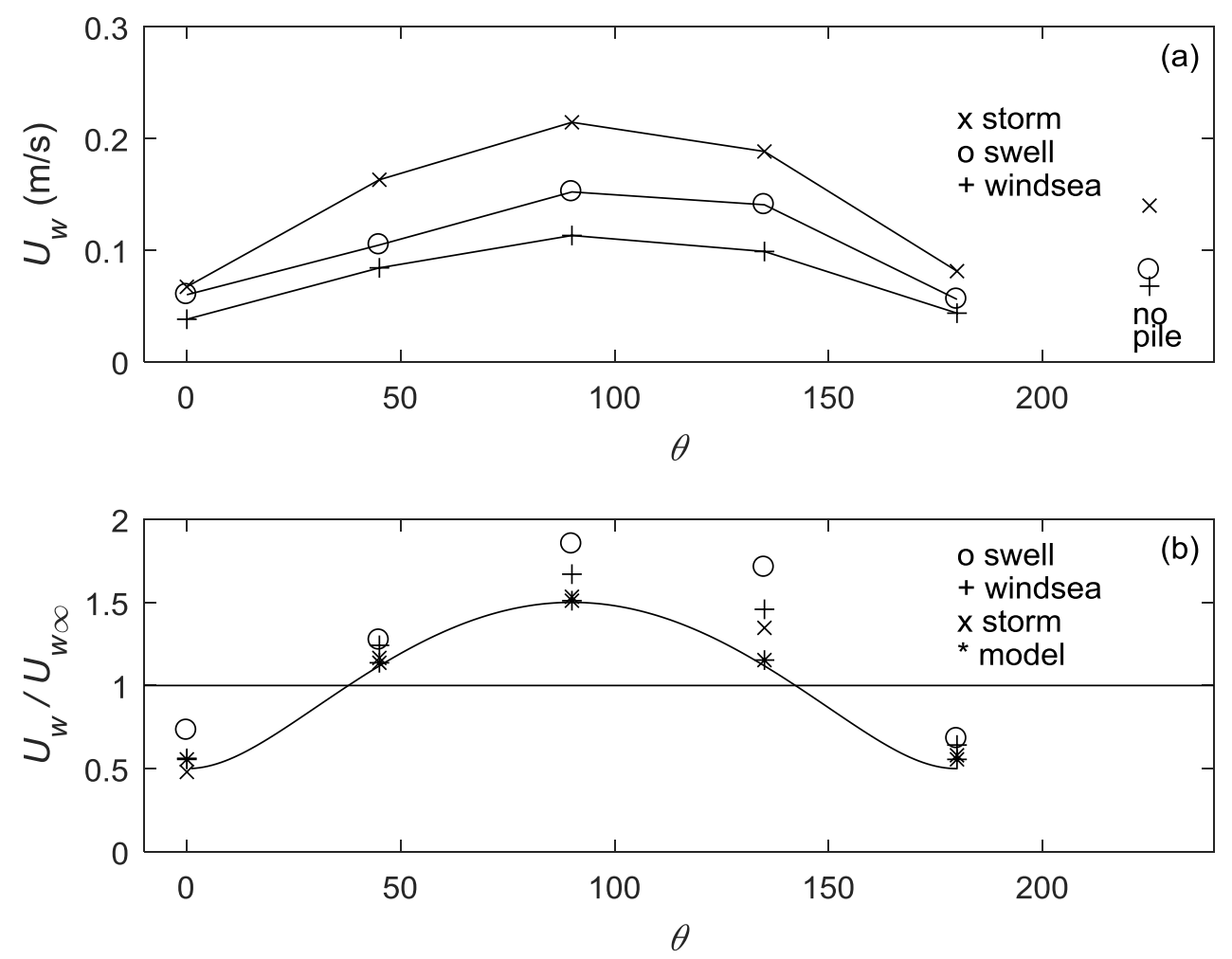

Fig. 25. (a) Orbital velocity $\left(U_{w}\right)$, at $z=0.025 \mathrm{~m}$ (scaled J-tube height), $\sim 0.75 \mathrm{D}$ from pile centre, varying with position around the pile, in modelled windsea, swell, and storm conditions. (b) As for (a) but showing the enhancement in orbital velocity compared to background conditions. $0^{\circ}$ is up-wave, $90^{\circ}$ is to the side, and $180^{\circ}$ is down-wave.

Previous observations identify scour as a problem near monopile structures (e.g. Sumer et al., 2001; Whitehouse et al., 2011). The data here supports the idea that a key mechanism for this is the acceleration in flow around the sides of the pile, in line with the enhanced bed shear stresses identified by Sumer et al. (1997). Gradients in transport resulting from accelerated flow at the sides of the pile will also result in erosion towards the leading edge of the pile (Roulund et al., 2005). The influence of the pile on the mean flow in this data appears to extend much further than the scour region that might be expected, corresponding to the observations by Rees (2006).

The experiment $\mathrm{KC}$ numbers indicate that the relative magnitude of orbital excursion to pile diameter are similar to prototype conditions. Sumer et al. (1997) identified that it requires much larger KC numbers $(>6)$ before structures such as horseshoe vortices form under waves, and this would require either much longer wavelengths, or narrower pile diameters. The range of $\mathrm{D} / \lambda$ values here $(0.05$ to $0.9)$, in conjunction with the low KC numbers, put the data outside the range where Sarpkaya and Isaacson (1981) identified non-linear effects as being important. The lack of wave driven flow separation and associated turbulence may explain why the potential flow model does reasonably well in the wave only case, and why the down-wave recovery is relatively rapid.

Sumer et al. (2001) identified mechanisms by which the addition of waves to the current complicates the hydrodynamic picture. In steady flows, a turbulent wake region develops from where the boundary layer separates from the pile, and turbulence is therefore enhanced on the downstream side of the pile. In the case of currents only, data here shows that the flows on the 'stern' quarters are 
reduced. In the current + wave tests, the velocity on the down-current side is greater than on the upstream side, and this suggests that the waves are contributing to lateral mixing. It is therefore possible that in the orthogonal wave and current case, the downstream persistence of the velocity shadow may be less marked.

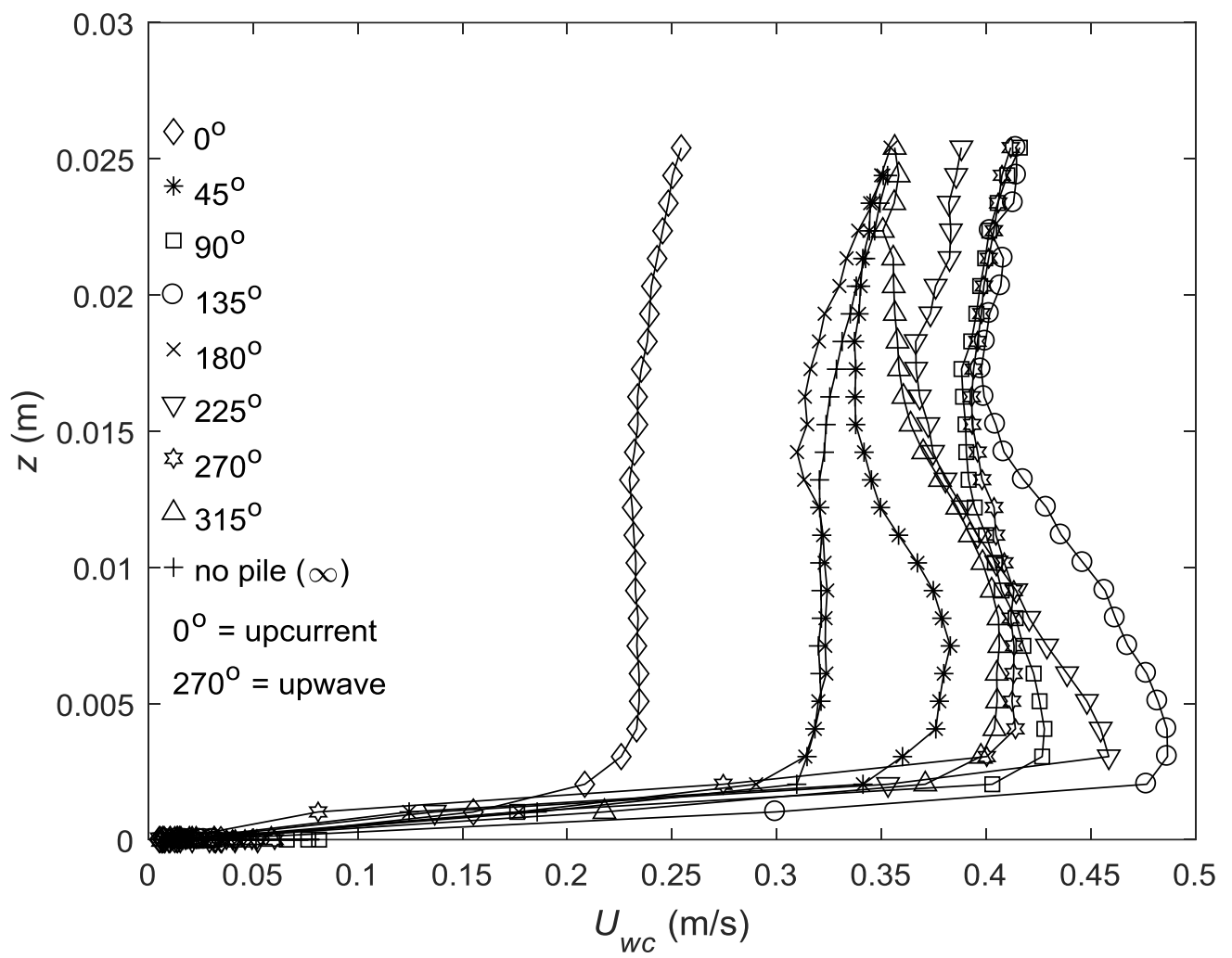

Fig. 26. Vertical profiles of resolved current plus wave orbital velocity maximum $\left(U_{w c}\right)$ for different positions around the pile. Zero to 180 degrees is the 'downwave' side of the pile.

The observations at the representative J-tube height suggest that just using the background flow conditions is not sufficient when considering the design requirements of cables. In these experiments, currents are enhanced by a factor of up to 1.35 , and the maximum enhancement happens at the sides of the monopile. Oscillatory velocities due to waves are enhanced by a factor of up to 1.85 when compared to the 'no-pile' case. The physical size of the model and the method of instrument deployment did not allow a particularly detailed near-pile mapping of the flows, and a larger model size would assist in this. Further, there is some difference between power cable take off design documented by Carter (2007), and that indicated by E.ON (pers. comm.), as might be expected for evolving technology and different installations. Parameterisations of flow near monopiles for this purpose therefore need flexibility to allow for different design options.

\section{Conclusions}

This study contributes measurements and parameterisations contributing to the quantification of the impact of monopile foundations on flow strengths and turbulence. 

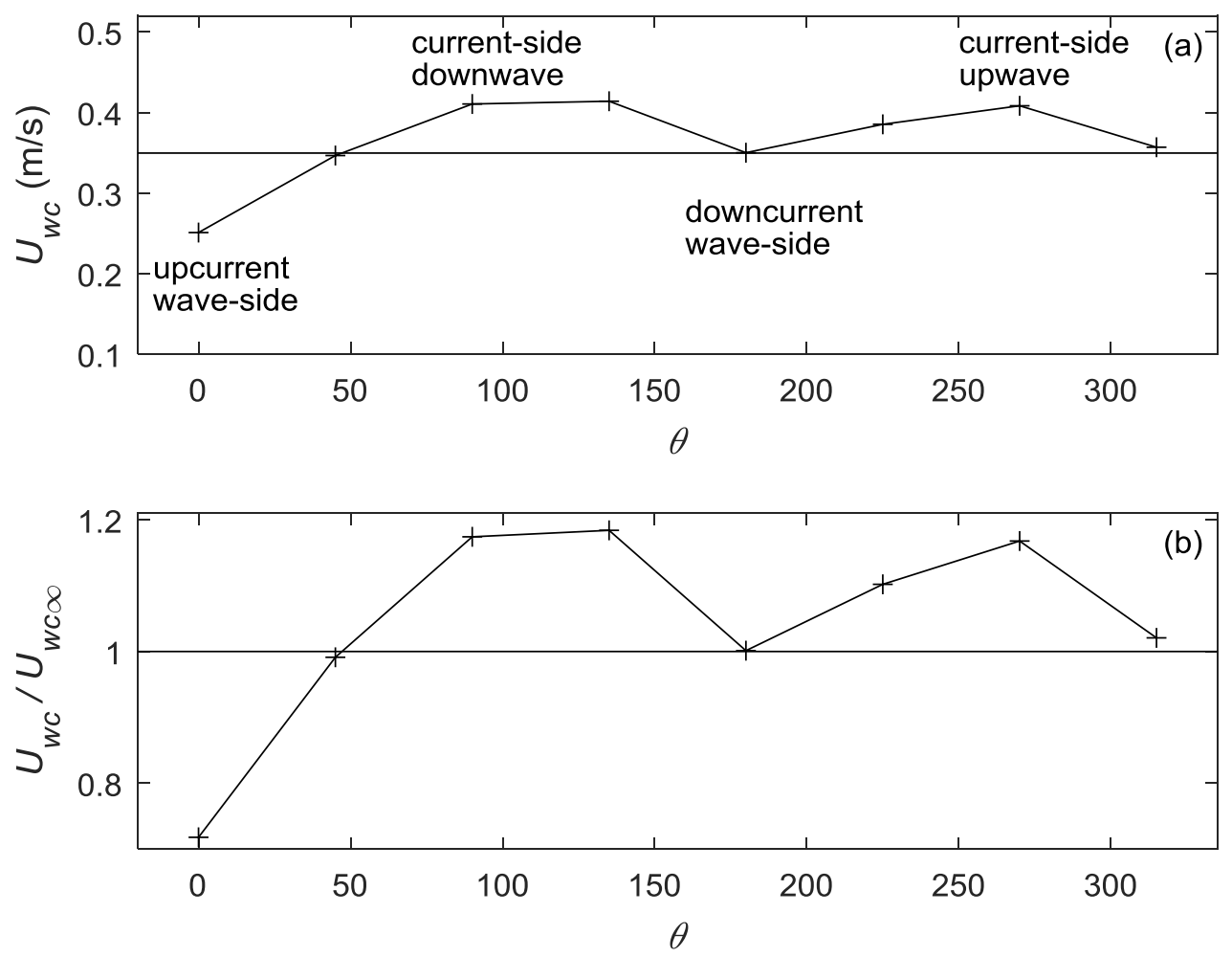

Fig. 27. (a) Variation in resolved current plus wave orbital velocity parameter $\left(U_{w c}\right)$ with position around the pile, at the height of the J-tube. $0^{\circ}$ is facing the flow (up-current). $270^{\circ}$ is facing the waves (up-wave). The horizontal line shows 'no pile' value in upper plot. (b) Enhancement from no-pile case.

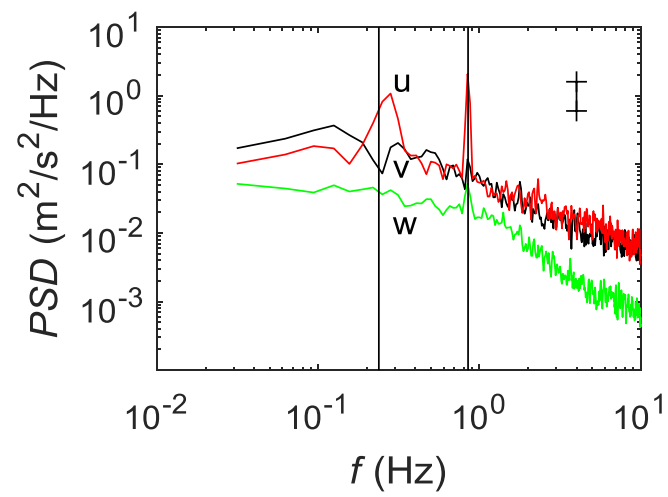

Fig. 28. Spectrum of velocity from the current+wave case, measured downstream of the pile (at $0.775 \mathrm{D}$ from the centre). The red $(u)$ spectrum is velocity in line with the wave direction, and is orthogonal to the current direction $(v)$. The peak at $0.85 \mathrm{~Hz}$ is at the wave frequency (vertical line), and there is a further peak, close to Strouhal frequency of $0.237 \mathrm{~Hz}$ (vertical line).

The mean flow was reduced immediately downstream of the pile, but returned to within $5 \%$ of background levels by $8.3 \mathrm{D}$ downstream of the pile centre in representative conditions. Turbulence 
peaked at 1.5 $\mathrm{D}$ from the pile, and the turbulent eddy shedding frequency was well predicted by the Strouhal number. Velocity magnitudes at the side of the pile were in line with potential flow theory and measured as up to 1.35 times greater than background flow rates. Velocities in the wake region were much less than predicted by potential flow theory, corresponding with increased turbulence in the wake.

Tests with waves indicated that oscillatory velocities reduced immediately down-wave of the pile, but returned to background levels within 1.65 to $3.5 \mathrm{D}$ from the pile centre. The general near-pile distribution of the orbital velocity maximum was well represented by potential flow theory. At the side of the pile in windsea conditions, the velocity increased up to 1.66 times the background level. This increased to 1.85 times in swell conditions.

For orthogonal currents and waves, and using the current direction as reference, maximum flows were observed at the side of the pile. These flows were enhanced by up to 1.2 times the no-pile case, at $0.75 \mathrm{D}$ from the pile centre. Peaks in the velocity spectra were evident at both wave frequency and at the Strouhal frequency, immediately down current from the pile.

\section{Acknowledgements}

Work towards this paper was part funded by the ERDF/European Union Interreg IVA program as part of the OFELIA project (Offshore Foundations Environ-mentaL Impact Assessments) (ref 5709/4295). The assistance of Dr Dave Simmonds, Professor Gregorio Iglesias and the Plymouth University COAST facility team is gratefully acknowledged.

\section{References}

Acheson, D.J., 1990. Elementary Fluid Dynamics. Oxford University Press, Oxford.

Baker, C. J., 1979. The laminar horseshoe vortex. Journal of Fluid Mechanics, 95, 347-367.

Carter, J.M.F., 2007. North Hoyle Offshore Wind Farm: design and build. Proceedings of the Institution of Civil Engineers, Energy, 160 (EN1), 21-29.

CEFAS, 2006. Scroby Sands Offshore Wind Farm - Coastal Processes Monitoring. Final Report AEO262 prepared for Marine Environmental Division, Defra and Department of Trade and Industry.

Dargahi, B., 1989. The turbulent flow field around a circular cylinder. Experiments in Fluids, 8, 1-12.

Jenkins, G.M. and Watts, D.G., 1968. Spectral analysis and its applications. Holden Day, San Fransisco, Ca.

Kassab, J.Y., 1989. Experimental design and statistical analysis. University College of North Wales Centre for Applied Statistics.

Masselink, G., Austin, M., O'Hare, T., Russell, P., 2007. Geometry and dynamics of wave ripples in the nearshore of a coarse sandy beach. Journal of Geophysical Research, 112, C1, 0022, doi:10.1029/2006JC003839.

Matutano, C., Negro, V., López-Gutiérrez, J.S., Esteban, M.D., Hernandez, A., 2014. The effect of scour protections in offshore wind farms. Journal of Coastal Research, SI 70, doi: 10.2112/SI70-003.1.

May, R.W.P., Ackers, J.C., Kirby, A.M., 2002. Manual on scour at bridges and other hydraulic structures, CIRIA, London, England.

Nezu, I., Nakagawa, H., 1993. Turbulence in open channel flows. Balkema, Rotterdam, the Netherlands.

Nielsen, A.W., Sumer, B.M., Fredsøe, J., Christensen, E.D., 2011. Sinking of armour layer around a cylinder exposed to a current. Proceedings of the ICE-Maritime Engineering, 164(4), 159-172.

Oppenheim, A.V., Schafer, R.W., 1975. Digital signal processing, Prentice Hall, New Jersey.

Petersen, T.U., Sumer, B.M., Fredsøe, J., Raaijmakers, T.C., Schouten. J.J., 2015. Edge scour at scour protections around piles in the marine environment - Laboratory and field investigations. Coastal Engineering 106, 42-72, doi: 10.1016/j.coastaleng.2015.08.007 0378-3839.

Rees, J. 2006. The impact of offshore windfarms on coastal processes- Scroby scour pits and scour wakes. Proceedings of the $30^{\text {th }}$ International Conference on Coastal Engineering, San Diego. Paper 392. 
Rivier, A., Bennis, A.C., Pinon, G., Gross, M., Magar, V., 2014. Regional numerical modelling of offshore monopile wind turbine impacts on hydrodynamics and sediment transport. Proceedings $1^{\text {st }}$ International Conference on Renewable Energies Offshore, Lisbon.

Rivier, A., Bennis, A.C., Pinon, G., Magar, V., Gross, M., 2016. Parameterization of wind turbine impacts on hydrodynamics and sediment transport. Ocean Dynamics. DOI 10.1007/s10236-016-0983-6.

Rogan, C., Miles, J., Simmonds, D., Iglesias, G., 2014. The Hydrodynamics of Monopile Foundations Experimental Measurements of Near Bed and Free Stream Turbulence. Proceedings $1^{\text {st }}$ International Conference on Renewable Energies Offshore, Lisbon.

Rogan, C., Miles, J., Simmonds, D., Iglesias, G. 2016. The Turbulent Wake of a Monopile Foundation. Renewable Energy 93. 180-187, doi:10.1016/j.renene.2016.02.050

Roulund, A., Sumer, B.M., Fredsoe, J., Michelson, J. 2005. Numerical and experimental investigation of flow and scour around a circular pile. Journal of Fluid Mechanics, 534, 351-401

Sarpkaya, T., Isaacson, M., 1981. Mechanics of wave forces on offshore structures. Van Nostrand Reinhold Company, New York.

Sumer, B.M., Christiansen, N., Fredsoe, J., 1997. The horseshoe vortex and vortex shedding around a vertical wall mounted cylinder exposed to waves. Journal of Fluid Mechanics, 332, 41-70.

Sumer, B.M., Fredsøe, J., Christiansen, N., 1992. Scour around vertical pile in waves. ASCE Journal of Waterway, Port, Coastal and Ocean Engineering, 118 (1), 15-31.

Sumer, B.M., Whitehouse, R.J., Torum, A., 2001. Scour around coastal structures: a summary of recent research. Coastal Engineering, 44 (2), 153-190.

Vanhellemont, Q., Ruddick, K., 2014. Turbid Wakes Associated with Offshore Wind Turbines Observed with Landsat 8. Remote Sensing of Environment, 145, 105-115.

Vattenfall, 2011. Kentish Offshore Wind Farm Extension Draft Environmental Statement Sections 5: Project Definition. Vattenfall, March 2011.

White, F.M., 1991. Viscous Fluid Flow. McGraw-Hill, In., New York.

Whitehouse, R.J.S., Harris, J.M., Sutherland, J., Rees, J., 2008. An assessment of field data for scour at offshore wind turbine foundations. Proceedings $4^{\text {th }}$ International Conference on Scour and Erosion, Tokyo.

Whitehouse, R.J.S., Harris, J.M., Sutherland, J., Rees, J., 2011. The nature of scour development and scour protection at offshore windfarm foundations. Marine Pollution Bulletin, 62, 73-88. 